University of Wollongong

Research Online

\title{
Using Tetracysteine-Tagged TDP-43 with a Biarsenical Dye To Monitor Real-Time Trafficking in a Cell Model of Amyotrophic Lateral Sclerosis
}

\author{
Janice S. W Ng \\ University of Cambridge \\ Maya A. Hanspal \\ University of Cambridge \\ Naunehal S. Matharu \\ University of Cambridge \\ Teresa P. Barros \\ University of Cambridge \\ Elin K. Esbjorner \\ Chalmers University of Technology
}

See next page for additional authors

Follow this and additional works at: https://ro.uow.edu.au/ihmri

Part of the Medicine and Health Sciences Commons

\section{Recommended Citation}

Ng, Janice S. W; Hanspal, Maya A.; Matharu, Naunehal S.; Barros, Teresa P.; Esbjorner, Elin K.; Wilson, Mark R.; Yerbury, Justin J.; Dobson, Christopher M.; and Kumita, Janet R., "Using Tetracysteine-Tagged TDP-43 with a Biarsenical Dye To Monitor Real-Time Trafficking in a Cell Model of Amyotrophic Lateral Sclerosis" (2019). Illawarra Health and Medical Research Institute. 1473.

https://ro.uow.edu.au/ihmri/1473

Research Online is the open access institutional repository for the University of Wollongong. For further information contact the UOW Library: research-pubs@uow.edu.au 


\title{
Using Tetracysteine-Tagged TDP-43 with a Biarsenical Dye To Monitor Real-Time Trafficking in a Cell Model of Amyotrophic Lateral Sclerosis
}

\author{
Abstract \\ TAR DNA-binding protein 43 (TDP-43) has been identified as the major constituent of the proteinaceous \\ inclusions that are characteristic of most forms of amyotrophic lateral sclerosis (ALS) and ubiquitin \\ positive frontotemporal lobar degeneration (FTLD). Wild type TDP-43 inclusions are a pathological \\ hallmark of $>95 \%$ of patients with sporadic ALS and of the majority of familial ALS cases, and they are \\ also found in a significant proportion of FTLD cases. ALS is the most common form of motor neuron \\ disease, characterized by progressive weakness and muscular wasting, and typically leads to death within \\ a few years of diagnosis. To determine how the translocation and misfolding of TDP-43 contribute to ALS \\ pathogenicity, it is crucial to define the dynamic behavior of this protein within the cellular environment. It \\ is therefore necessary to develop cell models that allow the location of the protein to be defined. We \\ report the use of TDP-43 with a tetracysteine tag for visualization using fluorogenic biarsenical \\ compounds and show that this model displays features of ALS observed in other cell models. We also \\ demonstrate that this labeling procedure enables live-cell imaging of the translocation of the protein from \\ the nucleus into the cytosol.

\section{Disciplines} \\ Medicine and Health Sciences

\section{Publication Details} \\ Ng, J. S. W., Hanspal, M. A., Matharu, N. S., Barros, T. P., Esbjorner, E. K., Wilson, M. R., Yerbury, J. J., \\ Dobson, C. M. \& Kumita, J. R. (2019). Using Tetracysteine-Tagged TDP-43 with a Biarsenical Dye To \\ Monitor Real-Time Trafficking in a Cell Model of Amyotrophic Lateral Sclerosis. Biochemistry, 58 (39), \\ 4086-4095.

\section{Authors} \\ Janice S. W Ng, Maya A. Hanspal, Naunehal S. Matharu, Teresa P. Barros, Elin K. Esbjorner, Mark R. \\ Wilson, Justin J. Yerbury, Christopher M. Dobson, and Janet R. Kumita
}




\section{Using tetracysteine-tagged TDP-43 with a biarsenical dye to monitor real-time trafficking in a cell model of Amyotrophic Lateral Sclerosis}

Janice S. W. $\mathrm{Ng}^{1 * *}$, Maya A. Hanspal ${ }^{1 * *}$, Naunehal S. Matharu ${ }^{1}$, Teresa P. Barros ${ }^{1}$, Elin K. Esbjörner $^{2}$, Mark R. Wilson ${ }^{3,4}$, Justin J. Yerbury,4, Christopher M. Dobson ${ }^{3}$ and Janet R. Kumita ${ }^{*}$

${ }^{1}$ Centre for Misfolding Diseases, University of Cambridge, Lensfield Road, Cambridge, CB2 $1 \mathrm{EW}$, UK; ${ }^{2}$ Department of Biology and Biological Engineering, Division of Chemical Biology, Chalmers University of Technology, Kemivägen 10, 41296 Gothenburg, Sweden; 3lllawarra Health and Medical Research Institute, Wollongong, NSW, Australia; ${ }^{4}$ Molecular Horizons and

School of Chemistry and Molecular Bioscience, Faculty of Science Medicine and Health, University of Wollongong, Northfields Avenue, Wollongong, NSW, 2522, Australia.

Running Title: Real-time imaging of TDP-43 trafficking in ALS

${ }^{* *}$ Authors contributed equally

*To whom correspondence should be addressed: Janet R. Kumita: Centre for Misfolding

Diseases, University of Cambridge, Lensfield Road, Cambridge, CB2 1EW, UK, irk38@cam.ac.uk, Tel. +44(0)1223761480 


\begin{abstract}
TAR DNA-binding protein 43 (TDP-43) has been identified as the major constituent of the proteinaceous inclusions that are characteristic of most forms of amyotrophic lateral sclerosis (ALS) and ubiquitin positive frontotemporal lobar degeneration (FTLD). Wild type (WT) TDP-43 inclusions are a pathological hallmark of $>95 \%$ of patients with sporadic ALS and of the majority of familial ALS cases, and they are also found in a significant proportion of FTLD cases. ALS is the most common form of motor neurone disease, characterised by progressive weakness and muscular wasting, and typically leads to death within a few years of diagnosis. To determine how the translocation and misfolding of TDP-43 contributes to ALS pathogenicity, it is crucial to define the dynamic behaviour of this protein within the cellular environment. It is therefore necessary to develop cell models that allow the location of the protein to be defined. We report the use of TDP-43 with a tetracysteine tag for visualisation using fluorogenic biarsenical compounds, and show that this model displays features of ALS observed in other cell models. We also demonstrate that this labelling procedure enables live-cell imaging of the translocation of the protein from the nucleus into the cytosol.
\end{abstract}




\section{Introduction}

Amyotrophic lateral sclerosis (ALS) is a progressive, and ultimately fatal, neurodegenerative disorder that primarily affects the upper and lower motor neurons of the central nervous system (CNS). Symptoms of the disease are normally first observed at a focal site of onset and then gradually spread to contiguous regions of the nervous system over time. As is the case with a number of other neurodegenerative diseases, this process is accompanied by the deposition of insoluble inclusions of aggregated protein in the cytoplasm of affected cell types which acts as a pathological signature for the condition. A major component of these inclusions has been identified as hyperphosphorylated and ubiquitinated pathological forms of both full-length and proteolytic cleavage fragments of TDP-43 1-3. Interestingly, scores of additional proteins are found co-deposited in spinal motor neurons with various forms of TDP-43 4.

Under physiological conditions, TDP-43 is a predominantly nuclear protein and, amongst other functions, it is involved in mRNA regulation and splicing ${ }^{1-3,5}$. In ALS, however, it has been found to mislocalise to the cytoplasm where it forms misfolded aggregates. The formation of preinclusions that do not associate with ubiquitin ${ }^{6}$ coincides with the movement of TDP-43 from the nucleus, and is considered to be an early aggregated species that precedes the accumulation of mature, ubiquitin-associated inclusions 6,7. There is great debate surrounding the relative contributions to pathogenesis of the loss of functional TDP-43 from the nucleus (loss-offunction, LOF) and of the accumulation of aggregated species with toxic properties in the cytoplasm (gain-of-function, GOF), although recent evidence suggests that both mechanisms can contribute to the disease ${ }^{8}$. Indeed, cytoplasmic translocation and aggregation of TDP-43 are directly associated with cell death, suggesting that the study of the initial stages of these processes could significantly increase our understanding of how TDP-43 contributes to the molecular pathology of the disease.

Fluorescent labels have been employed in many live cell models to allow the observation of the intracellular distribution of proteins. The tetracysteine (TC) motif and biarsenical dye system makes use of a derivative of fluorescein, called fluorescein arsenical hairpin binder (FIAsH). This dye binds to a short amino acid sequence with the TC-motif, having the general structure Cys-Cys-Xaa-Xaa-Cys-Cys (CCXXCC, in which X denotes any amino acid) ${ }^{9}$. Both FIAsH and a red-shifted variant, $\mathrm{ReAsH}$, denoting resorufin arsenical hairpin binder, are commercially available and well characterised fluorescent dyes. These reagents are not fluorescent in their unbound states, but interaction with the TC motif results in a large increase in their fluorescence 
quantum yields. Previous studies to optimise the flanking regions of the TC peptide sequence resulted in two 12-amino acid motifs, FLNCCPGCCMEP and HRWCCPGCCKTF, with high capacities to accommodate the FIAsH and ReAsH dyes ${ }^{10}$. Such optimised TC tags can be genetically inserted into the target protein, allowing the $\mathrm{FIAsH}$ or $\mathrm{ReAsH}$ dye to bind with high specificity. A significant advantage of the TC tag compared to the use of fluorescent proteins is that its small size reduces the likelihood of it significantly affecting the properties of the protein of interest ${ }^{11}$. This system lends itself to the visualisation of the target protein inside live cells because of the relatively low cytotoxicity of the dye ${ }^{12}$. Furthermore, it has been shown that a variety of proteins in common mammalian cell lines, primary cortical neurons and also Gramnegative bacteria can be labelled with $\mathrm{FIAsH}$ and $\mathrm{ReAsH}{ }^{13}$. Both reagents have been used successfully to report on the conformation of proteins expressed in cultured cells ${ }^{14-16}$. In the context of the present study, we note that this labelling strategy has been used in cell models of several neurodegenerative diseases to investigate the conformational states of aberrantly misfolded proteins in a cellular environment ${ }^{17,18}$. For example, the incorporation of a TC tag into a variant of huntingtin ( $\mathrm{Htt}_{\mathrm{ex} 1}$ ) has been used to probe the aggregation state of the protein in Neuro2A cells, in which the conformational properties of the monomeric form but not the aggregated forms, enable the TC tag to bind to ReAsH ${ }^{17}$. In addition, transfected SH-SY5Y cells have been used to overexpress TC-labelled a-synuclein, allowing the dynamics and structural properties of the aggregates to be studied using in situ microscopy techniques including fluorescence recovery after photobleaching (FRAP) and confocal fluorescence anisotropy ${ }^{18}$.

We discuss in this paper the development of a live cell model of ALS using transiently transfected SH-SY5Y cells overexpressing TC tagged WT TDP-43 (HA-TDP43-TC) that allows direct visualisation of the protein in living cells using the biarsenical dye $\mathrm{FIAsH}$. We demonstrate that the TC tag does not detectably alter the behaviour of TDP-43 in this cell model by comparing it to cells overexpressing HA-TDP-43 (HA-TDP43), a human influenza haemagglutinin (HA) epitope tagged TDP-43 which is a well-characterised system used previously to investigate the role of TDP-43 in ALS ${ }^{19,20 . ~ W e ~ s h o w ~ h e r e ~ t h a t ~ t h e ~ H A-T D P 43-T C ~}$ model recapitulates key biochemical features of TDP-43 proteinopathies, such as association with stress granule markers and phosphorylation 19, 21, 22. Furthermore, we show it is possible to monitor noninvasively, and with spatiotemporal resolution, the cytoplasmic accumulation of TDP-43 concomitantly with its nuclear clearance over the course of $72 \mathrm{~h}$ post-transfection. Finally, we demonstrate that this model is amenable to time-lapse confocal microscopy, and 
observe FIAsH labelled TDP-43 transferring from the nucleus into the cytoplasm in real time.

\section{Materials and Methods}

\section{Construction of plasmids}

To generate the HA- and TC-tagged WT TDP-43 (HA-TDP43 and HA-TDP43-TC) sequences, the cDNA encoding TDP-43 was amplified from the pCMV.SPORT6.1_TDP-43 plasmid (Source BioScience, Nottingham, UK) by PCR using Phusion High-fidelity DNA polymerase (Thermo Fisher Scientific, Loughborough, UK) and primers that either incorporated the DNA sequences of the HA-tag and the TC-tag (TDP43-TC (forward) and TDP43-TC (reverse) primers) or which incorporated the HA-tag only (HA-TDP43 (forward) and HA-TDP43 (reverse)). Sequences of the primers are reported in Table S1 and the DNA sequences encoding HA-TDP43 and HA-TDP43TC are shown in Figure S1. In both cases, the primers introduced unique BamHI and Xbal sites at the 5' and 3' ends of the coding sequences respectively. These sites were used to clone the genes of interest into the pcDNA3. $1^{\mathrm{TM}}(+)$ vector (Thermo Fisher Scientific). The constructs were transformed using standard heat-shock protocols into chemically competent $\mathrm{DH} 5 \mathrm{a}^{\mathrm{TM}} E$. coli (Thermo Fisher Scientific) and plated on LB-agar plates containing ampicillin (100 $\mu \mathrm{g} / \mathrm{mL})$. DNA was isolated using a Qiaprep Spin mini-prep or maxi-prep kit (Qiagen, Manchester, UK) according to the manufacturer's instructions. Purified DNA concentrations were determined using a NanoDrop® ND-1000 Spectrophotometer (Thermo Fisher Scientific) and constructs were confirmed by DNA sequencing (Department of Biochemistry, University of Cambridge, UK).

\section{Cell culture and transfection}

The human neuroblastoma SH-SY5Y cell line was routinely cultured in complete growth medium (DMEM/F12 supplemented with $10 \%(\mathrm{v} / \mathrm{v}) \mathrm{FBS}$ ) in a humidified chamber at $37^{\circ} \mathrm{C}$ in $95 \%$ air and $5 \% \mathrm{CO}_{2}$. Cells were either chemically transfected using Lipofectamine ${ }^{\circledR} 2000$ (Thermo Fisher Scientific) or electroporated using the Neon ${ }^{\mathrm{TM}}$ system (Thermo Fisher Scientific).

\section{Lipofection}

Cells were plated one day before transfection in $35 \mathrm{~mm}$ glass bottom dishes (Ibidi, ThistleScientific, Glasgow, UK) such that the culture was at $70-90 \%$ confluence on the day of transfection. Transfections were carried out using Lipofectamine ${ }^{\circledR} 2000$ according to the manufacturer's instructions in serum free media (DMEM/F12 without FBS). Briefly, the 
plasmid/Lipofectamine ${ }^{\circledR} 2000$ complex was prepared at a 1:4 ratio ( $\left.\mu \mathrm{g}: \mu \mathrm{L}\right)$. Lipofectamine ${ }^{\circledR} 2000$ was incubated (room temperature (RT), $5 \mathrm{~min}$ ) in half the total volume of media before being mixed with the media containing the plasmid DNA, followed by further incubation (RT, $20 \mathrm{~min}$ ). The complete media was aspirated from the cultured cells and replaced with the DNA/Lipofectamine complex in serum free media. The cells were incubated in the DNA/Lipofectamine-containing media $\left(37^{\circ} \mathrm{C} .5 \mathrm{~h}\right)$ which was then replaced with complete media, and the cells were incubated overnight at $37^{\circ} \mathrm{C}$ before being used for further experiments.

\section{Electroporation}

Two days prior to electroporation, cells were seeded into T-75 flasks with complete growth media such that the cells were $70-90 \%$ confluent on the day of the experiment. The cells were then detached with $0.25 \%$ Trypsin-EDTA (3 mL, Thermo Fisher Scientific) and washed in phosphate buffered saline, $\mathrm{pH} 7.4$ (PBS). The cells were re-suspended in the resuspension buffer provided and electroporated $(1,100 \mathrm{~V}, 50 \mathrm{~ms}, 1 \mathrm{X})$ according to the manufacturer's protocol (Neon Transfection System, Thermo Fisher Scientific). After electroporation, the cells were seeded in complete growth media in 6-well, or 96-well plates (Corning, Appleton Woods Limited, Birmingham, UK), or in $35 \mathrm{~mm}$ glass bottom dishes (Ibidi, Thistle Scientific), and left for at least $24 \mathrm{~h}$ before use in experiments.

\section{Immunocytochemistry}

SH-SY5Y cells overexpressing TDP-43 were grown in $35 \mathrm{~mm}$ glass bottomed dishes. To monitor the effects over time, immunocytochemistry was performed with cells at 24, 48 and $72 \mathrm{~h}$ post transfection. The cells were washed with chilled PBS and fixed with chilled paraformaldehyde (3.7\% in PBS, $15 \mathrm{~min}$ ). After $15 \mathrm{~min}$, the cells were washed twice with chilled PBS (10 min) and permeabilised by incubating in chilled PBS-T (0.3\% (v/v) Triton-X in PBS, 30 $\mathrm{min})$. After $30 \mathrm{~min}$, the cells were blocked with BSA (5\% (w/v) in PBS-T, $30 \mathrm{~min})$ and washed twice with chilled PBS (5 min). The cells were incubated with the primary antibody (1:1000) (RT for $1 \mathrm{~h}$ or $4{ }^{\circ} \mathrm{C}$ overnight) with gentle rocking. For co-localisation experiments, cells were coincubated with the primary antibodies anti-HA 3F10 (Sigma Aldrich UK Ltd., Gillingham UK) and either phospho (403/404) TDP-43 (ProteinTech, Manchester, UK) or phospo (409/410) TDP43 (ProteinTech), or TIA-1 C-20 (Santa Cruz Biotechnology, Heidelberg, Germany). This was followed by incubation with an appropriate secondary Alexa Fluor ${ }^{\mathrm{TM}}$ antibody (1:1000, Life Technologies, Paisley, UK) (RT for $1 \mathrm{~h}$ or $4^{\circ} \mathrm{C}$, overnight). Wheat germ agglutinin (WGA) Alexa Fluor $^{\mathrm{TM}} 647$ conjugate (Life Technologies) treatment was done prior to permeabilisation by 
treating the cells with a 1:500 dilution of WGA conjugate in Hank's buffered salt solution (HBSS; $10 \mathrm{~min}, \mathrm{RT})$. Cells were washed twice in HBSS and permeabilisation was performed with subsequent labelling. Cells were washed twice with chilled PBS after incubation with each of the primary and secondary antibodies. After washing, the cells were incubated with the nuclear stain Hoechst ( $1 \mu \mathrm{g} / \mathrm{mL}$, Life Technologies) for $5 \mathrm{~min}$, and the labelled cells were mounted with Ibidi mounting medium (Ibidi, Thistle Scientific). Imaging of the samples was performed on a Leica TCS SP8 Confocal Microscope (Leica Microsystems, Wetzlar, Germany) at the Cambridge Advanced Imaging Centre (CAIC), University of Cambridge.

\section{In-cell biarsenical dye labelling}

For the labelling of TC tagged TDP-43 (HA-TDP43-TC) overexpressed in SH-SY5Y cells, the TC-FIAsH ${ }^{\mathrm{TM}}$ II In-Cell Tetracysteine Tag Detection Kit from Molecular Probes (Thermo Fisher Scientific) was used. Transfected SH-SY5Y cells were grown in $35 \mathrm{~mm}$ glass bottomed dishes. To monitor the effects over time, in-cell FIAsH labelling was performed at 24, 48 and $72 \mathrm{~h}$ post transfection. The cells were washed twice with reduced serum Opti-MEM ${ }^{\circledR}$ without phenol red (Life Technologies), and incubated with FIAsH $(1 \mu \mathrm{M})$ in $\mathrm{Opti}_{-\mathrm{MEM}^{\circledR}}\left(37^{\circ} \mathrm{C}, 30 \mathrm{~min}\right.$, protected from light). After $30 \mathrm{~min}$, the cells were washed twice with $1 \mathrm{X}$ BAL (2, 3-dimercaptopropanol) buffer in Opti-MEM ${ }^{\circledR}\left(37^{\circ} \mathrm{C}, 1 \mathrm{~h}\right.$, protected from light). For live-cell imaging at each time point, cells were incubated with Hoechst stain $(1 \mu \mathrm{g} / \mathrm{mL})$ in Opti-MEM ${ }^{\circledR}$ for 5 min after the BAL buffer wash steps. Finally, the cells were washed with Opti-MEM ${ }^{\circledR}$ and cultured in Opti-MEM ${ }^{\circledR}$ and prepared for live-cell imaging.

\section{Quantification of transfection efficiency}

Cells were transfected with either HA-TDP43 or HA-TDP43-TC using Lipofectamine ${ }^{\circledR} 2000.24 \mathrm{~h}$ post-transfection, the cells were fixed and immunostained with an anti-HA antibody and an AlexaFluor 488 secondary antibody, to detect positively transfected cells, and Hoechst nuclear counterstain, to visualise the total number of cells. The samples were imaged using a Leica TCS SP5 confocal microscope equipped with 40x HPC Fluo Tar and 63x APO oil immersion objectives, and a UV diode and $488 \mathrm{~nm}$ argon laser line to visualise the Hoechst and AlexaFluor 488 fluorescence respectively (Leica Microsystems, Milton Keynes, UK). Three images were obtained at 40x magnification in different regions of the dish for each sample. Subsequent quantification was performed using ImageJ, using the Cell Counter plugin to count manually all cells stained with the anti-HA antibody or Hoechst stain. The number of cells positive for both anti-HA and Hoechst were calculated as a percentage of the total number of Hoechst positive 
cells.

\section{Time-lapse imaging}

Cells transfected with HA-TDP43-TC in $35 \mathrm{~mm}$ glass dishes were labelled with the Molecular Probes $^{\mathrm{TM}}$ FIAsH-EDT 2 dye from Invitrogen ${ }^{\mathrm{TM}}$ Life Technologies ${ }^{\mathrm{TM}}$, 24-36 $\mathrm{h}$ post-transfection. Cells were washed in Opti-MEM ${ }^{\circledR}(\mathrm{RT}, 2 \mathrm{X})$ followed by incubation in FIAsH-EDT 2 (1 $\mu \mathrm{M}$ in Opti$\left.M M^{\circledR}\right)(R T, 30$ min, protected from light). The cells were washed twice in 1 X BAL buffer (in Opti-MEM $\left.{ }^{\circledR}\right)\left(37^{\circ} \mathrm{C}, 1 \mathrm{~h}\right.$, protected from light) and imaged in Opti-MEM ${ }^{\circledR}$. The chamber slide placed in a CO2-UNIT-BL Stage top heated $\mathrm{CO}_{2}$ chamber $\left(37^{\circ} \mathrm{C}\right.$, no $\mathrm{CO}_{2}$ perfusion) 30 min prior to imaging, using confocal microscopy (Leica Microsystems). The inbuilt software (LAS AF, Leica Microsystems) was used for time-lapse imaging using the 'Best Focus' function at different locations within the dish using the 'Mark and Find' function (overnight, 15 min intervals).

\section{Quantification of depletion of nuclear fluorescence from time-lapse images}

Each frame of the time-lapse video was analysed as an individual tiff file in ImageJ, and analysis was performed in the green channel of each image. A region of interest was drawn around the nucleus of the target cell exhibiting fluorescence using the freeform tool. 'Set measurements' was selected from the Analyze menu, and 'area', 'integrated density' and 'mean gray value' were ticked. 'Measure' was selected from the 'Analyze' menu to obtain values. The process was repeated for an area of the image without fluorescence to measure the background signal, and was performed for all frames up to $11 \mathrm{~h}$ of imaging, after which there was no difference between nuclear fluorescence and the background signal. The results were copied and pasted into Microsoft Excel for further analysis. Equation 1 was used to obtain a value for corrected fluorescence at each time point.

Equation 1:

integrated intensity - (area of region of interest $\times$ mean background)

Comparison of the cellular location of TDP-43 in HA-TDP43 and HA-TDP43-TC following electroporation treatment

Confocal images acquired from cells expressing HA-TDP43 and HA-TDP43-TC after labelling with an anti-HA antibody were used to determine the location of fluorescence signals by dividing into three categories: (1) predominantly nuclear localisation, (2) localisation in both cytoplasm 
and nucleus and (3) predominantly cytoplasmic localisation. Fifty cells were randomly counted from different sample preparations and the percentage was calculated for each category.

\section{Immunoprecipitation experiments}

Transfected cells were rinsed with PBS $(10 \mathrm{~mL})$ and detached using a trypsin-EDTA solution followed by the addition of complete growth medium to neutralise the trypsin. The cells were pelleted by centrifugation $\left(4^{\circ} \mathrm{C}, 5 \mathrm{~min}, 1500 \mathrm{rpm}\right)$ and the supernatant was removed. The cell pellet was washed with chilled PBS and further centrifuged $\left(4^{\circ} \mathrm{C}, 5 \mathrm{~min}, 1500 \mathrm{rpm}\right)$. The supernatant was removed, and the cell pellet was lysed with RIPA buffer $(1 \mathrm{~mL}, 50 \mathrm{mM}$ Tris$\mathrm{HCl}, \mathrm{pH}$ 8.0, $150 \mathrm{mM}$ sodium chloride, 1.0\% Igepal CA-630 (NP-40), 0.5\% sodium deoxycholate, $0.1 \%$ sodium dodecyl sulphate) supplemented with EDTA-free protease inhibitor cocktail (Roche Diagnostics, Mannheim, Germany) for $10 \mathrm{~min}$ on ice, followed by centrifugation $\left(4^{\circ} \mathrm{C}, 10 \mathrm{~min}, 10,000 \mathrm{rpm}\right)$, after which the supernatant was retained for immunoprecipitation. Pierce $^{\mathrm{TM}}$ Anti-HA Magnetic Beads (Thermo Fisher Scientific, $25 \mu \mathrm{L}$ ) were washed twice with TBS-T (Tris-buffered saline, $0.05 \%(\mathrm{v} / \mathrm{v})$ Tween $20,300 \mu \mathrm{L}$ ), followed by a final wash with $\mathrm{ddH}_{2} \mathrm{O}$; with brief vortexing between washes. The cell lysate $(1 \mathrm{~mL})$ was added to a $1.5 \mathrm{~mL}$ microfuge tube followed by addition of the pre-washed magnetic beads. The sample was then mixed and incubated (RT, $30 \mathrm{~min}, 200 \mathrm{rpm}$ ) with constant rotation. After incubation, the tube was placed into a DynaMag ${ }^{\text {TM }}$-Spin Magnet stand (Thermo Fisher Scientific) and the supernatant containing unbound protein was collected and saved for analysis. To elute, the HAtagged TDP-43 bound to the magnetic beads, Pierce HA Peptide (Thermo Fisher Scientific) $(100 \mu \mathrm{L}$ of $2 \mathrm{mg} / \mathrm{mL}$ ) was added to the bead slurry, and this was then vortexed and incubated $\left(37^{\circ} \mathrm{C}, 10 \mathrm{~min}\right)$. The eluted sample was then analysed by Western blotting.

\section{Western Blotting}

SDS-PAGE was used to separate protein samples prior to western blotting. The protein sample $(20 \mu \mathrm{L})$ was prepared in NuPAGE® LDS Sample Buffer (4X) (LifeTechnologies) and NuPAGE® Sample Reducing Agent (10X) (Life Technologies) and run on a NuPAGE® 4-12 \% gradient Bis-Tris (Life Technologies) gel with MES running buffer (Life Technologies) (200 V, $25 \mathrm{~min}$ ). The proteins were transferred from the gel to an iBlot $\AA^{\circledR}$ Transfer Stack with PVDF membrane (0.2 $\mu \mathrm{m}$ pore size) using the $\mathrm{iBlot} \circledast$ Dry Blotting System (Life Technologies) according to the manufacturer's protocol. After transfer, the PVDF membrane was blocked with 5\% (w/v) dry skimmed milk powder in PBS with $0.05 \%$ Tween-20 (blocking buffer) (RT, $1 \mathrm{~h}$, gentle agitation). The membrane was then incubated with primary antibodies in blocking buffer $\left(1: 1000,4^{\circ} \mathrm{C}\right.$, 
overnight or RT, $1 \mathrm{~h}$ ) followed by washing four times with PBS-T (RT, $10 \mathrm{~min}$ ). After washing, the membrane was incubated with AlexaFluor 488- or 594-labelled secondary antibody (1:1000, RT, $1 \mathrm{~h}$ ). After incubation, the membrane was washed four times with PBS-T and imaged using a Typhoon 9400 laser-based scanner (GE Healthcare) at $550 \mathrm{~V}$ using a green (532 nm) excitation laser to excite AlexaFluor 594 or a blue $(488 \mathrm{~nm})$ laser to excite AlexaFluor 488.

\section{Results}

Transient transfection of SH-SY5Y cells to overexpress full length TDP-43 containing a Cterminal tetracysteine-tag (TC)

A number of TDP-43 cell models use epitope tags or fluorescent fusion proteins to distinguish between overexpressed TDP-43 and the endogenous protein 7, 19, 23-25. For our model, we added the 12 amino acid TC tag (FLNCCPGCCMEP) ${ }^{10}$ to the C-terminus of full-length TDP-43 (Figure 1 , i). It has been established that in disease states such as ALS, C-terminal truncated fragments of TDP-43 are found in the inclusions ${ }^{3,26}$, and so we reasoned that the addition of the TC tag to the C-terminus of our full-length TDP-43 construct will allow us to visualise both the full-length and truncated fragments of TDP-43. We also incorporated an HA-epitope tag at the N-terminus of the protein (Figure 1, ii) in the same construct to allow an alternative means of identification using antibody detection 19, 23, 25, 27, 28. The HA-TDP43-TC construct was inserted into the mammalian expression vector, pcDNA3.1(+). Despite the tags introduced being of relatively small sizes $(\sim 1 \mathrm{kDa})$, we checked that the addition of the $\mathrm{C}$-terminal TC tag did not change the cellular behaviour of the overexpressed WT TDP-43, by comparing our TC-tagged TDP-43 to an $\mathrm{N}$-terminally HA-tagged full-length TDP-43 (HA-TDP43, Fig. 1, iii) construct which has been previously shown to have diffuse nuclear localisation in transiently transfected cells 19,20 .

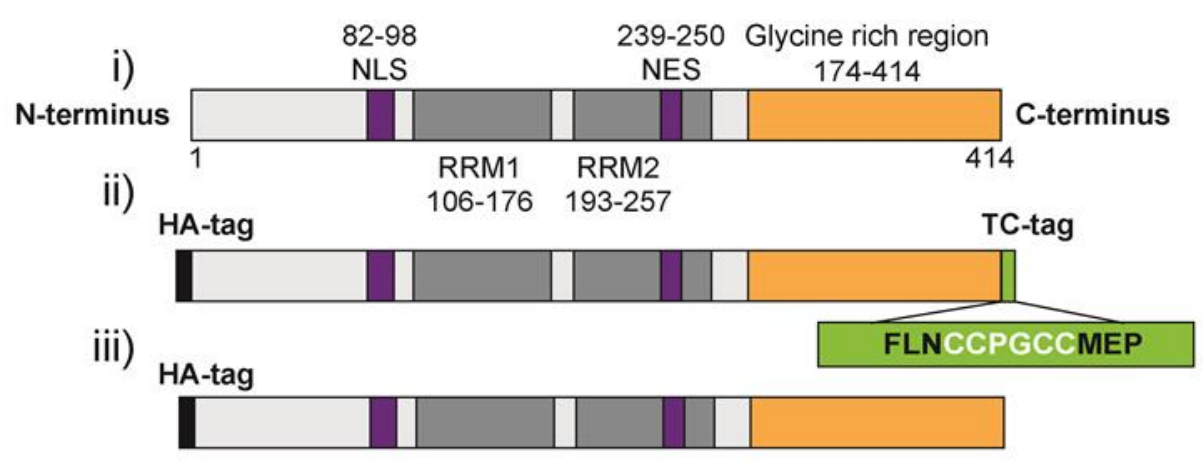

Figure 1. Schematic diagram illustrating i) full length wild-type TDP-43, ii) HA-TDP43-TC and iii) HA-TDP43 constructs. The HA-tag is denoted in black, the nuclear localisation signal (NLS) and nuclear export signal (NES) are shown in purple, the RNA-recognition motifs are shown in dark 
grey, the glycine rich region is orange and the TC-tag is shown in green.

The HA-TDP43 and the HA-TDP43-TC constructs were transiently transfected into SH-SY5Y cells using two methods: lipofection (Lipofectamine ${ }^{\circledR} 2000$ ) and electroporation (Neon ${ }^{\mathrm{TM}}$ system) to determine which most efficiently delivers the HA-TDP43-TC plasmid into the cells. SH-SY5Y cells are a human neuroblastoma cell line that is well-established and extensively used in neurodegenerative disease models, including for studies involving TDP-43 18, 19, 25, 29, 30. We used this cell line for the current study as they are consistently amenable to transient transfection protocols and have a favourable nucleus-to-cytoplasm ratio, which allows clear microscopy imaging. After $24 \mathrm{~h}$ post transfection, the cells were fixed, permeabilised and probed with an anti-HA primary antibody. For both the HA-TDP43 and HA-TDP43-TC expressing cells, HA-specific labelling was detected as predominantly diffuse nuclear fluorescence (Figure 2A). By analysing the number of positively stained cells for the two different transfection methods, we observed a $10 \%$ and $12 \%$ transfection efficiency for the HA-TDP43 and HA-TDP43-TC plasmids, respectively, using the lipofection method, and a $14 \%$ transfection efficiency for both plasmids with the electroporation method (Table S2). For the HA-TDP43-TC cell line, we could image the live cells $24 \mathrm{~h}$ post-transfection using the FIAsH dye (Figure $2 \mathrm{~B}$ ), finding that FIAsHbound protein was predominantly localised to the nucleus. It is interesting to note that for the lipofected cells, the FIAsH-bound HA-TDP43-TC displayed a weaker signal intensity compared to electroporated cells, although this was not statistically significant $(n=1$, two-tailed unpaired $t$ test, $p=0.1404$ ) (Table S3). We proceeded with the electroporation method in light of its greater efficiency, using it to characterise the intracellular distribution of HA-TDP43-TC over time. To confirm further the presence of the full-length protein within the cells $24 \mathrm{~h}$ post-transfection, the HA-tagged proteins were isolated using immunoprecipitation (anti-HA magnetic beads) and the resulting samples were analysed by Western blotting (Figure 2C); similar results were observed for the $48 \mathrm{~h}$ and $72 \mathrm{hr}$ post-transfected samples (data not shown). 
A
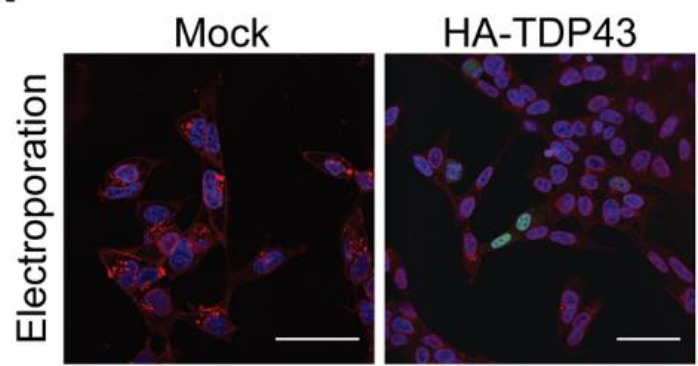

HA-TDP43-TC
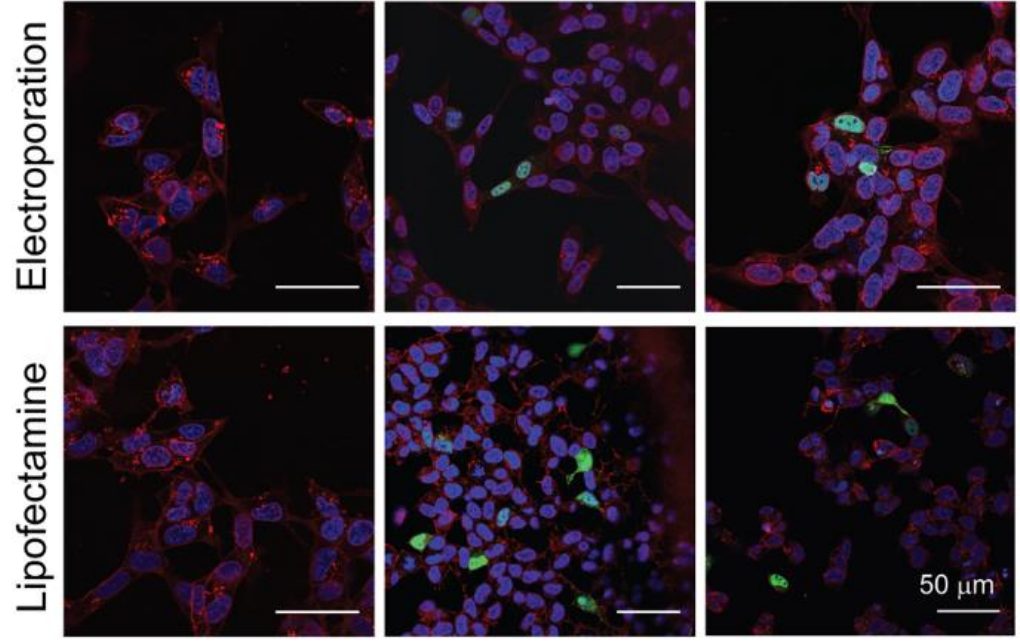

B

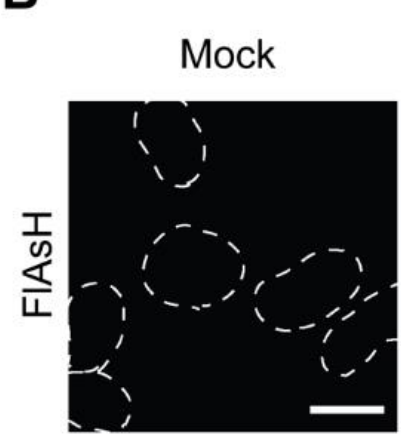

HA-TDP43-TC Electroporation

HA-TDP43-TC Lipofectamine
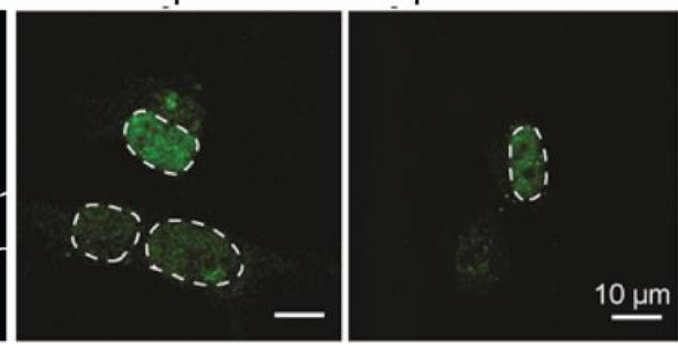

C

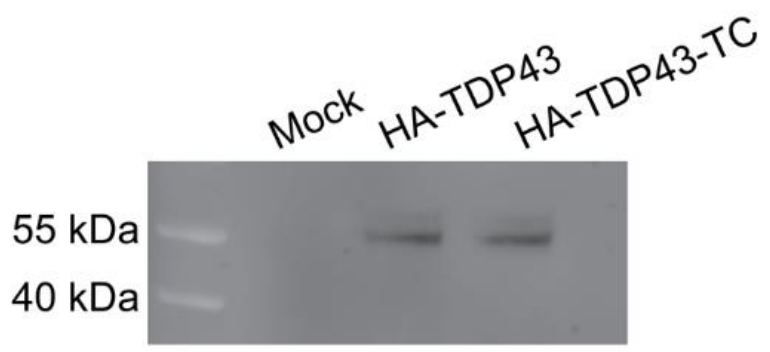

Figure 2. Confocal images of SH-SY5Y cells transfected (by either electroporation or lipofection) to overexpress HA-TDP43 or HA-TDP43-TC, at $24 \mathrm{~h}$ post-transfection. A) immunofluorescence images generated using an anti-HA antibody and an AlexaFluor 488 secondary antibody (green), Hoechst nuclear stain (blue) and membranes are stained with wheat germ agglutinin (WGA) Alexa Fluor ${ }^{\mathrm{TM}} 647$ conjugate (red), scale bars are $50 \mu \mathrm{m}$; B) fluorescence images after the addition of the FIAsH dye (24 h post-transfection). For clarity, the white dotted line denotes the nucleus. Images are representative of multiple independent experiments. C) Immunoprecipitation followed by Western blot analysis of HA-TDP43 and HATDP43-TC isolated from SH-SY5Y cell lysates at $24 \mathrm{~h}$ post transfection. Mock transfections are cells transfected with buffer alone. 


\section{Monitoring HA-TDP43-TC expression at different time points post-transfection with the}

FIAsH dye

In previously reported cell models, WT TDP-43 is overexpressed and remains localised within the nucleus ${ }^{19,31}$, whereas disease-related variants show translocation of the protein from the nucleus to the cytoplasm where they form inclusions when monitored up to $72 \mathrm{~h}$ post transfection. When cells expressing WT TDP-43 are exposed to conditions of stress, such as by the addition of arsenite or through nutrient deprivation, this aberrant behaviour is also observed 21, 22. To determine whether or not FIAsH labelling was able to identify HA-TDP-43-TC positively over $72 \mathrm{~h}$ post transfection, we compared live-cell images after the addition of the FIAsH reagent to HA-TDP43-TC expressing cells at 24,48 and $72 \mathrm{~h}$ post-transfection. In Neontransfected SH-SY5Y cells expressing HA-TDP43-TC, we observed diffuse nuclear staining 24 h post-transfection (Figure 3, $24 \mathrm{~h}$ ). At $48 \mathrm{~h}$ post-transfection, however, FIAsH fluorescence was observed in both the nucleus and the cytoplasm (Figure 3, $48 \mathrm{~h}$ ), and by $72 \mathrm{~h}, \mathrm{FIAsH}$ labelled HA-TDP43-TC was predominantly in the cytoplasm and appeared as inclusions of variable size (Figure 3, $72 \mathrm{~h}$ ). The cells with strong staining of cytoplasmic inclusions showed a lack of nuclear fluorescence. No FIAsH fluorescence was observed at any time point (24-72 $h$ ) when the over-expressed protein lacked the TC tag (Figure S2). Translocation of the HA-TDP43-TC protein had clearly occurred spontaneously in this cell model despite earlier reports that in the absence of exogenous stress, WT TDP-43 remains in the nucleus ${ }^{32-34}$. We next examined whether this translocation was due to the C-terminal TC tag perturbing the behaviour of WT TDP-43 protein or if the mode of transfection had itself imposed an exogenous stress. To carry out this objective we used the well-characterised HA-TDP43 cell model ${ }^{19}$ and compared results from time course experiments in which cells were transfected using either electroporation or lipofection. 

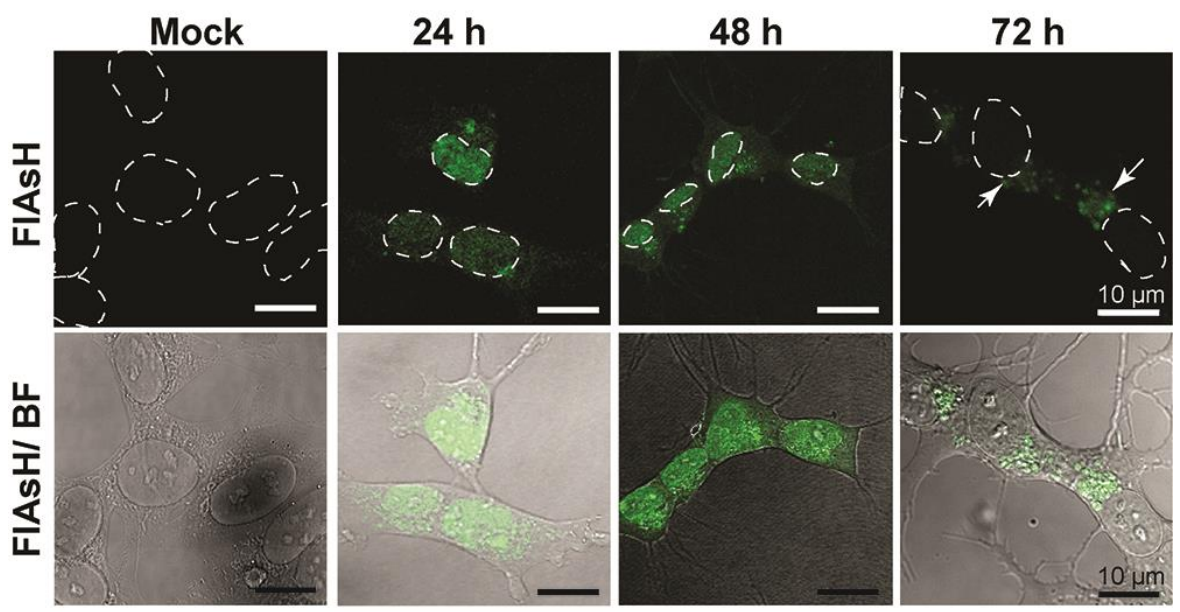

Figure 3. Confocal images of FIAsH bound HA-TDP43-TC in live SH-SY5Y cells at 24, 48 and $72 \mathrm{~h}$ post transfection. FIAsH fluorescence images only (upper panel), or merged with bright field (BF) images (lower panel). The images are representative of multiple independent experiments. For clarity, the outline of the nuclei is indicated by dashed white lines. The white arrowheads indicate punctate staining of HA-TDP43-TC. Mock transfection is cells transfected with buffer alone.

SH-SY5Y cells were transfected to express HA-TDP43 using electroporation and, similarly to HA-TDP43-TC, translocation of the protein was observed at 48 and $72 \mathrm{~h}$ post-transfection (Figure 4A and 4B). Comparison of the number of cells containing nuclear or cytoplasmic TDP43 (detected through HA-tag labelling, $n=50$ cells per condition) showed that the HA-TDP43 and the HA-TDP43-TC models both displayed similar distributions (Figure 4C \& 4D). When the cells underwent lipofection however, much less translocation was observed in both cell models and at $72 \mathrm{~h}$ post-transfection the nuclear TDP-43 staining remained prominent (Figure S3), in agreement with previous studies $20,24,25,27,28$. Interestingly, when the electroporation method was used to transiently transfect cells to overexpress EGFP-tagged TDP-43, we found that at $48 \mathrm{~h}$ and $72 \mathrm{~h}$ post-transfection, the TDP43-EGFP fluorescence was predominantly localised in the nucleus (Figure S4). This predominant nuclear localisation by WT TDP43-EGFP at $48 \mathrm{~h}$ and $72 \mathrm{~h}$ post-transfection has been reported previously 35,36 . 

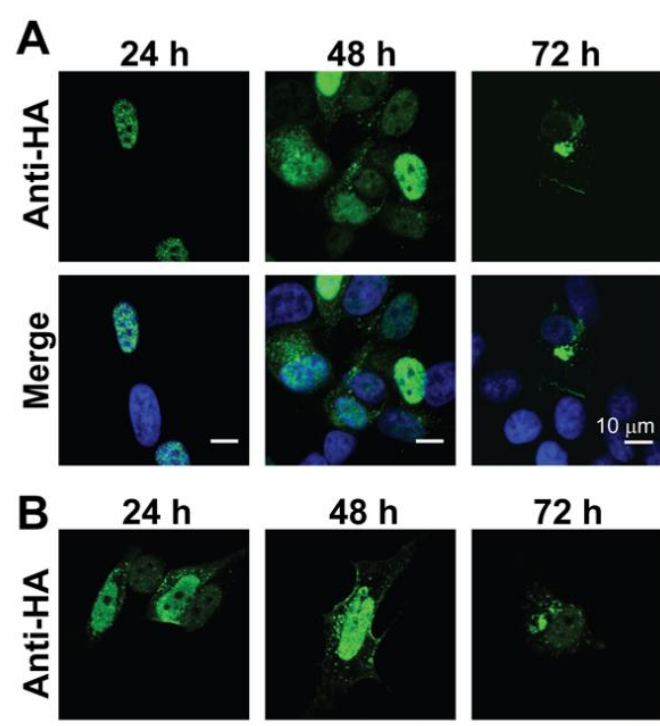

$48 \mathrm{~h}$

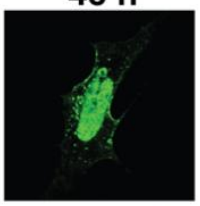

$72 \mathrm{~h}$
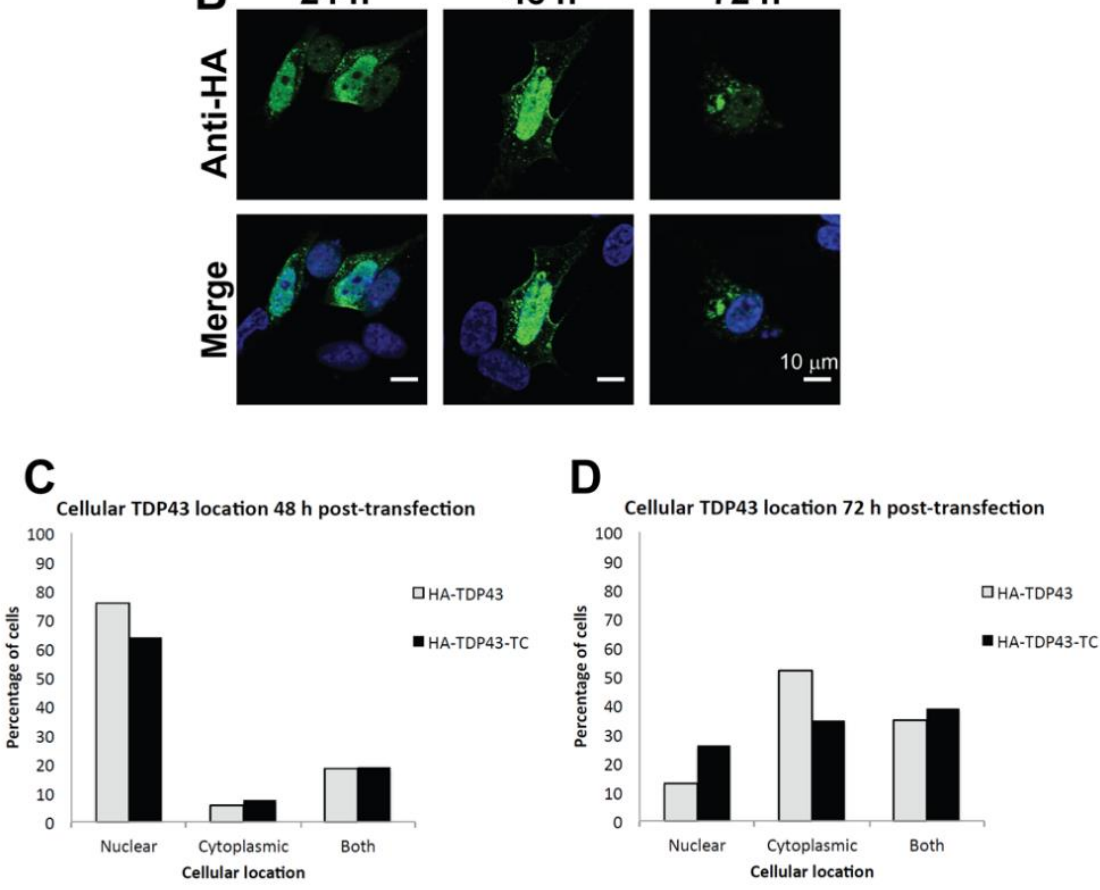

Figure 4. HA-TDP43 and HA-TDP43-TC expression and distribution in SH-SY5Y cells at 48 and $72 \mathrm{~h}$ following transfection by electroporation $\mathrm{A})$ Representative images of cellular TDP-43 distribution for HA-TDP43 (A) and HA-TDP43-TC (B); scale bars are $10 \mu \mathrm{m}$. Merged immunofluorescence images generated using an anti-HA antibody (green) and Hoechst nuclear counterstain (blue). Histograms showing cellular location of HA-TDP43 and HA-TDP43-TC at (C) $48 \mathrm{~h}$ and (D) $72 \mathrm{~h}$ post-transfection. Percentages were calculated by examining cells with positive HA-tag labelling from 8 independent imaging experiments until 50 cells for each condition ( $A$ and $B$ ) were identified.

\section{HA-TDP43-TC shows co-localisation with the stress granule marker TIA-1}

To determine if the cytoplasmic inclusions observed in cells expressing HA-TDP43-TC provide a useful model of ALS pathogenesis, we next examined whether or not the protein had colocalised with a marker of stress granules (SGs), dynamic RNA-containing complexes that are formed in the cytoplasm when cells are subjected to stress ${ }^{37}$. SGs direct translation towards 
proteins required for cell survival and repair, but may under pathological conditions, stabilise and act as scaffolds to promote recruitment and aggregation of a variety of proteins, including TDP-43 38, 39. SGs in cell culture are formed within minutes of stress induction, and normally degrade within $3 \mathrm{~h}$ following the cessation of stress, but persist in response to chronic stress ${ }^{37}$. It has been reported that in a number of cell models, cytoplasmic TDP-43 aggregates are found to co-localise with stress granule markers such as TIA-1 in both cell culture models $22,32,40$ and immunocytochemical analyses of ALS and FTLD-TDP tissue samples 21, 22, 41. Chronic cellular stress may initiate pathological TDP-43 aggregation via its promotion of the assembly of SGs, thereby bringing together high concentrations of aggregation prone proteins and leading to cytoplasmic inclusion ${ }^{42}$. At 48 and $72 \mathrm{~h}$ post-transfection, we observed that cytoplasmic inclusions containing HA-TDP43-TC co-localised with TIA-1 (Figure 5); similar co-localisation was also observed in cells overexpressing HA-TDP43 (Figure 5). 


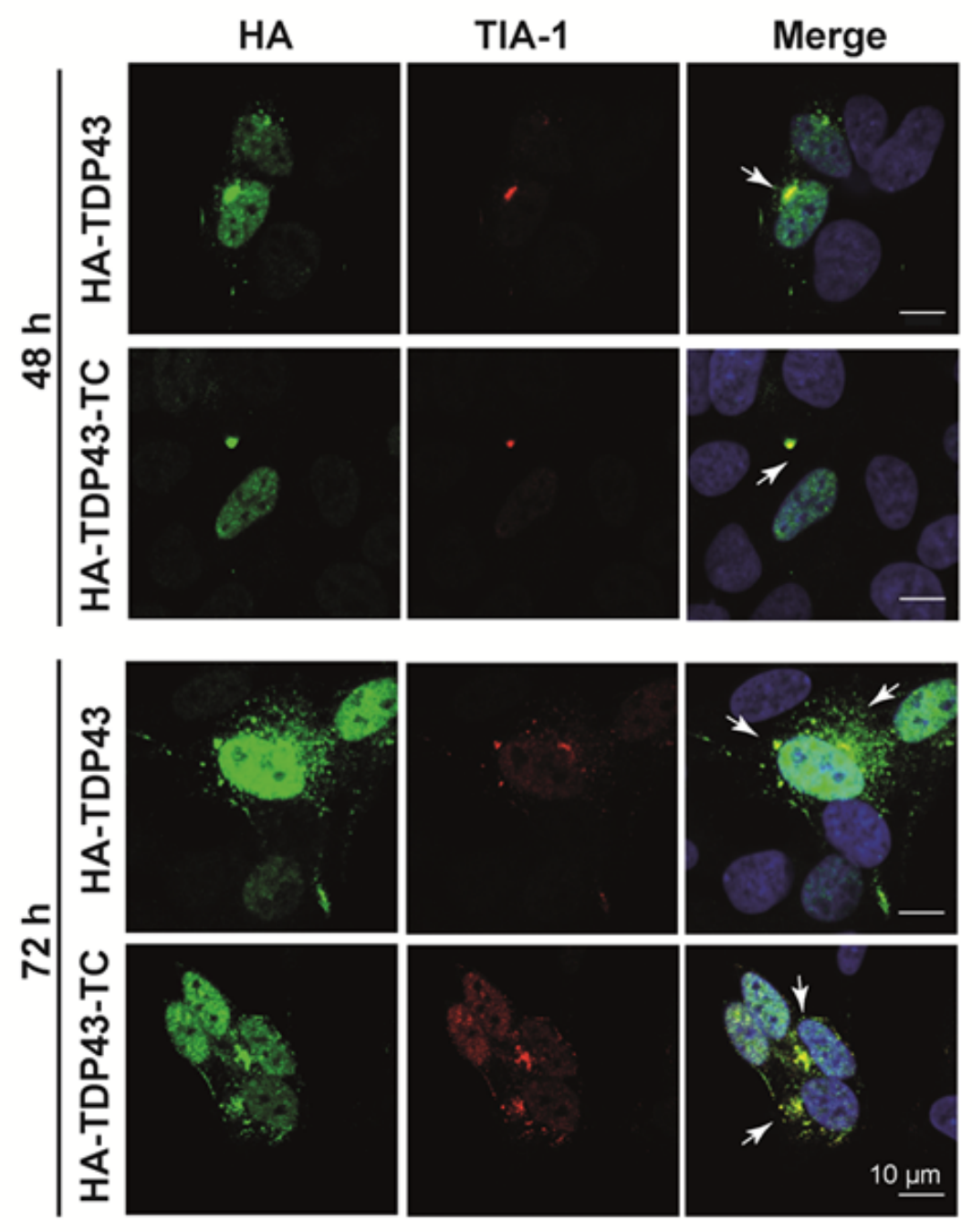

Figure 5. Comparison of the co-localisation of TDP-43 and the SG marker TIA-1 between the HA-TDP43 and HA-TDP43-TC SH-SY5Y cell models at $48 \mathrm{~h}$ and $72 \mathrm{~h}$ post transfection using electroporation. Merged immunofluorescence images generated using an anti-HA antibody (green) and Hoechst nuclear counterstain (blue) and anti-TIA-1 staining (red). Yellow denotes co-localisation between HA-labelled TDP-43 and TIA-1 (white arrows).

In addition to co-localisation with SG markers, phosphorylation of TDP-43 is commonly regarded as a hallmark feature of ALS. Using specific antibodies that recognise phosphorylated Ser403/404 or Ser409/410 epitopes, cytoplasmic inclusions containing phosphorylated TDP-43 have been detected in some cell models; this is not seen, however, in many cell-lines expressing WT TDP-43 36, 43. We probed transfected SH-SY5Y cells expressing HA-TDP43-TC with antibodies specific for TDP-43 phosphorylation at either Ser403/404 or Ser409/410. In SHSY5Y cells transfected to express HA-TDP43 or HA-TDP43-TC, at 48 and $72 \mathrm{~h}$ post transfection, no specific fluorescence was obtained using the pSer409/410 antibody (data not 
shown), but fluorescent cytoplasmic inclusions were detected using the pTDP-43 Ser403/404 antibody (Figure 6).

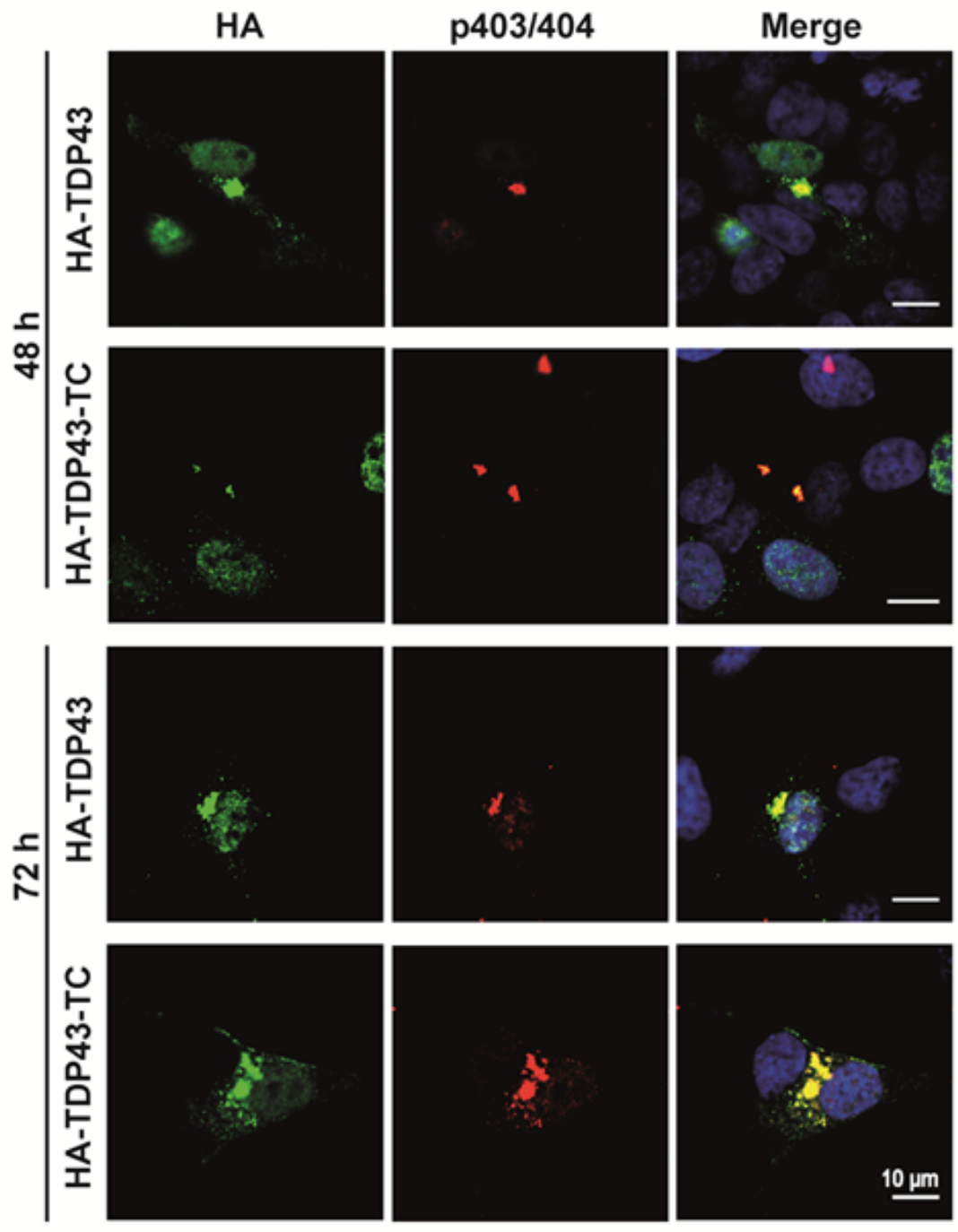

Figure 6. Comparison of co-localisation of TDP-43 using an antibody for detection of phosphorylation at serine 403 and 404 residues between the HA-TDP43 and HA-TDP43-TC $\mathrm{SH}-\mathrm{SY} 5 \mathrm{Y}$ cell models at $48 \mathrm{~h}$ and $72 \mathrm{~h}$ post transfection using electroporation. Merged immunofluorescence images generated using an anti-HA antibody (green), Hoechst nuclear counterstain (blue) and an anti-TDP-43 p403/404 antibody (red). Yellow denotes co-localisation between HA-labelled TDP-43 and TDP43-p403/404.

\section{The TC-tag cell model shows nuclear-to-cytoplasmic translocation in real-time}

Having demonstrated that the TC-tag cell model reproduces several events relevant to the disease process in ALS, we finally examined whether or not it is possible to monitor TDP-43 translocating from the nucleus into the cytoplasm in real-time. Given that the mild stress induced by electroporation appears to stimulate translocation of HA-TDP43-TC, no additional external 
chemicals or processes are needed to induce this process. At $24 \mathrm{~h}$ post-transfection, the FIAsH reagent was added to these cells and they were imaged using confocal microscopy for a further $20 \mathrm{~h}$ (Figure 7).

Initially, individual cells expressing HA-TDP43-TC show predominantly nuclear staining (Figure $7 \mathrm{a}$ ), but over the time-course of the experiment (Figure $7 \mathrm{~b}$ onwards) these cells can be seen to have developed small cytoplasmic inclusions. Interestingly, even at the initial time point when cytoplasmic TDP-43 can be visualised ( $26 \mathrm{~h} 30 \mathrm{~min}$ ), the fluorescence signal is not diffuse but appears as small puncta that clearly change position in relation to the cell nucleus over time. They also appear to coalesce during real-time imaging, suggesting that they merge into larger structures, or are just in close proximity to one another (Supplementary Video S1). The appearance of HA-TDP43-TC in the cytoplasm coincides with a gradual decrease in nuclear FIAsH signal intensity (Figure $7 \mathrm{c}$ onwards, Figure S5), indicating nuclear depletion of the labelled TDP-43. Concerns about the high background fluorescence due to endogenous cysteine-rich proteins and the potential cytotoxicity of biarsenical dyes have been raised ${ }^{44}$; however, with the application of BAL buffer washes, we did not observe high background fluorescence during our live-imaging studies, and the cells imaged did not show any signs of morphological change until late into the imaging process ( $44 \mathrm{~h}$, Figure $7 \mathrm{f}$ ), 


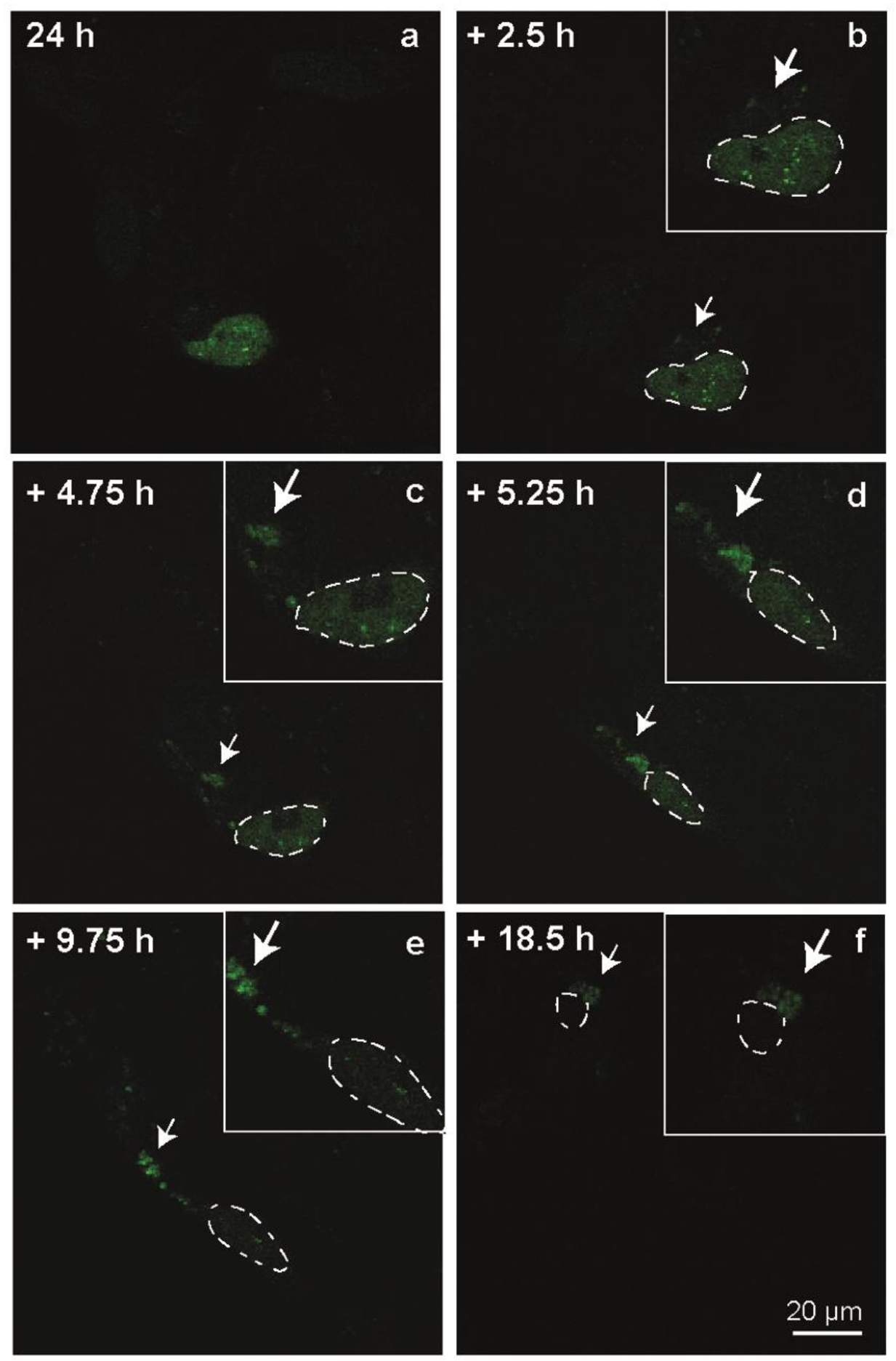

Figure 7. a-f. Frames from time-lapse confocal microscopy imaging of HA-TDP43-TC expressing SH-SY5Y cells labelled with FIAsH dye. Imaging commenced at $24 \mathrm{~h}$ posttransfection and the subsequent time points (h) shown are a) 24:00, b) 26:30, c) 28:45, d) 29:15, e) 33:45, and f) 42:30. The inset white boxes contain zoomed images of the cell nucleus (zoom factor $2 \mathrm{x}$ original image). The nucleus of the target cell is denoted by a dotted line and white arrows denote cytoplasmic puncta. The images are representative of multiple independent experiments. 


\section{Discussion}

Together with fluorescent protein (FP) tags, the HA tag is one of the most extensively used labels in investigations of the role of TDP-43 in ALS 19, 24, 45. The HA-tag, however, has a significant limitation, in that there is a need to fix and immunostain cells in order to detect the labelled TDP-43, therefore making this model incompatible with live cell imaging to monitor intracellular processes. The FP-TDP-43 fusion systems are amenable to live cell imaging, but involve the incorporation of a $\sim 27 \mathrm{kDa}$ FP. Given the relative size of the FP in relation to TDP43 ( 27 kDa versus $43 \mathrm{kDa}$ ), the bulkiness of the fluorescent moiety may perturb the native structure and function of the TDP-43 and therefore, the use of the smaller TC-tag in conjunction with biarsenical dyes ( 1 kDa) may prove advantageous.

In this paper we have described a cell model in which the cells are transiently transfected to overexpress HA-tagged TDP-43 with a C-terminal TC tag, which is visualised using the biarsenical dye, FIAsH. This model can be used to monitor the translocation of TDP-43 from the nucleus to the cytoplasm in live cells using time-lapse microscopy. Although our study shows that the HA-TDP43-TC model is very similar to the well-characterised HA-TDP43 cell model, the properties of the TC tag and FIAsH make it a very valuable tool for monitoring the intracellular distribution of TDP-43 in live cells. The TC tag itself is relatively small, while the FIAsH dye is membrane permeable and non-fluorescent until it binds to the TC tagged protein. In addition, the dye itself is sufficiently photostable, and shows low toxicity, to allow imaging in live cells over several hours ${ }^{13}$.

Comparisons between the HA-TDP43-TC and HA-TDP43 cell models indicate that the Cterminal TC tag does not detectably alter the properties of HA-TDP-43 when overexpressed in SH-SY5Y cells. When transfected by electroporation, at 48 and $72 \mathrm{~h}$ post-transfection, cells expressing either HA-TDP43 or HA-TDP43-TC both show cytoplasmic translocation and accumulation of the overexpressed protein. Interestingly, this observation differs from most previous reports in which WT HA-TDP43 or GFP-TDP43 has been overexpressed in transfected mammalian cell lines ${ }^{20,46}$. Nuclear to cytoplasmic translocation is more commonly observed in cell models expressing TDP-43 containing familial ALS mutations 47,48 or truncated forms of the protein 25,31 . For cells expressing WT TDP-43, translocation of the protein to the cytoplasm has generally been reported to occur only following the application of external stress ${ }^{21,32}$. For cells expressing HA-TDP43 or HA-TDP43-TC, we observe predominantly nuclear localisation when cells were lipofected, with much less cytoplasmic TDP-43 detected at all the time points 
measured post-transfection, than in cells transfected using electroporation. These results suggest that the electroporation method, which slightly increases the transfection efficiency (by $2-4 \%$, may also result in a degree of cell stress ${ }^{49}$; we therefore took advantage of this phenomenon to study the translocation of WT TDP-43 in the absence of pharmacological stressors.

Cells transfected by electroporation to express HA-TDP43-TC demonstrate some key biochemical hallmarks of ALS, including the nuclear to cytoplasm translocation, an increase in the appearance and size of cytoplasmic inclusions over time, an indication that the TDP-43 is at least partially phosphorylated and the association of the TDP-43 inclusions with TIA-1, a marker of stress granules 1, 3, 38. Phosphorylation of TDP-43 at the Ser403/404 or Ser409/410 epitopes has also been associated with ALS pathogenesis $43,50,51$. It is not clear whether phosphorylation of TDP-43 leads to aggregate formation and/or neurotoxicity, or if this process represents a normal reaction to the presence of an intracellular aggregate, as reports have shown that phosphorylation of WT-TDP43 is not always observed 20,24 . We did, however, observe positive staining with the antibody specific for p403/404 but not the antibody specific for p409/410 (Fig. $6)$.

Using cells expressing HA-TDP43-TC, we were able to observe translocation of WT TDP-43 in the absence of pharmacological stresses in live cells over time, as indicated by the appearance of fluorescent motile cytoplasmic puncta together with decreased nuclear fluorescence. This process is thought to precede the deposition of cytoplasmic inclusions, which may contribute to the pathogenesis of ALS due to depletion of TDP-43 in the nucleus, preventing the protein from carrying out its normal regulatory functions on mRNA (LOF), and/or to the accumulation of cytoplasmic TDP-43 with inherently toxic properties (GOF) ${ }^{52}$.

The HA-TDP43-TC cell model shares many features of the well-established HA-TDP43 model, which has been successfully utilised to investigate many aspects of TDP-43-mediated disease processes, but offers the considerable advantage of being compatible with live cell imaging. In particular, we were able to monitor the nuclear to cytoplasmic translocation of TDP-43 in live cells using confocal microscopy, which represents an early stage in the deposition of aggregated TDP-43 in the cytoplasm of affected cells.

Having shown that it is possible to image TDP-43 translocation in real-time using this model, it is 
clear that this has the potential to provide quantitative information about the translocation process itself, and could even be applied to gain further insights into other processes, which occur subsequently to nuclear translocation, such as the aggregation of TDP-43 within the intracellular environment. This in turn may help develop a better understanding of how TDP-43 contributes to neurodegeneration in ALS and related proteinopathies.

\section{Associated Content:}

\section{Supplementary Information.}

Supplementary text, tables and figures.

Supplementary time-lapse video related to Figure 7

\section{Accession Codes:}

TDP-43: Q13148-1

\section{Acknowledgements}

We would like to thank Dr. Kamran Yunus and Dr. Gabi Kaminski-Schierle for providing training and access to the confocal microscope (for live imaging) in the Department of Chemical Engineering and Biotechnology, University of Cambridge. We would like to thank Dr. Leila Luheshi for helpful discussions. J.S.W.N was supported by a Yousef Jameel Scholarship and The Cambridge Commonwealth, European \& International Trust, M.A.H. was supported by a University of Cambridge CHESS studentship and an Australian Government Endeavour Research Fellowship. The work was supported, in part, by the Wellcome Trust (094425/Z/10/Z; J.R.K., C.M.D.), the Centre for Misfolding Diseases (J.S.W.N., M.A.H., N.S.M., T.P.B., C.M.D., J.R.K.), the Wenner-Gren Foundations and the Swedish Research Council (grant 2016-03902, E.K.E.) and the NHMRC (grants 1084144 and 1095215, J.J.Y).

\section{Conflict of Interest}

The authors declare that they have no conflicts of interest with the contents of this article 


\section{References}

[1] Arai, T., Hasegawa, M., Akiyama, H., Ikeda, K., Nonaka, T., Mori, H., Mann, D., Tsuchiya, K., Yoshida, M., Hashizume, Y., and Oda, T. (2006) TDP-43 is a component of ubiquitinpositive tau-negative inclusions in frontotemporal lobar degeneration and amyotrophic lateral sclerosis, Biochem Biophys Res Commun 351, 602-611.

[2] Arai, T., Hasegawa, M., Nonoka, T., Kametani, F., Yamashita, M., Hosokawa, M., Niizato, K., Tsuchiya, K., Kobayashi, Z., Ikeda, K., Yoshida, M., Onaya, M., Fujishiro, H., and Akiyama, H. (2010) Phosphorylated and cleaved TDP-43 in ALS, FTLD and other neurodegenerative disorders and in cellular models of TDP-43 proteinopathy, Neuropathology 30, 170-181.

[3] Neumann, M., Sampathu, D. M., Kwong, L. K., Truax, A. C., Micsenyi, M. C., Chou, T. T., Bruce, J., Schuck, T., Grossman, M., Clark, C. M., McCluskey, L. F., Miller, B. L., Masliah, E., Mackenzie, I. R., Feldman, H., Feiden, W., Kretzschmar, H. A., Trojanowski, J. Q., and Lee, V. M. (2006) Ubiquitinated TDP-43 in frontotemporal lobar degeneration and amyotrophic lateral sclerosis, Science 314, 130-133.

[4] Ciryam, P., Lambert-Smith, I. A., Bean, D. M., Freer, R., Cid, F., Tartaglia, G. G., Saunders, D. N., Wilson, M. R., Oliver, S. G., Morimoto, R. I., Dobson, C. M., Vendruscolo, M., Favrin, G., and Yerbury, J. J. (2017) Spinal motor neuron protein supersaturation patterns are associated with inclusion body formation in ALS, Proc Natl Acad Sci U S A 114, E3935-E3943.

[5] Buratti, E., and Baralle, F. E. (2001) Characterization and functional implications of the RNA binding properties of nuclear factor TDP-43, a novel splicing regulator of CFTR exon $9, \mathrm{~J}$ Biol Chem 276, 36337-36343.

[6] Farrawell, N. E., Lambert-Smith, I. A., Warraich, S. T., Blair, I. P., Saunders, D. N., Hatters, D. M., and Yerbury, J. J. (2015) Distinct partitioning of ALS associated TDP-43, FUS and SOD1 mutants into cellular inclusions, Sci Rep 5, 13416, 1-14.

[7] Smethurst, P., Newcombe, J., Troakes, C., Simone, R., Chen, Y. R., Patani, R., and Sidle, K. (2016) In vitro prion-like behaviour of TDP-43 in ALS, Neurobiol Dis 96, 236-247.

[8] Cascella, R., Capitini, C., Fani, G., Dobson, C. M., Cecchi, C., and Chiti, F. (2016) Quantification of the Relative Contributions of Loss-of-function and Gain-of-function Mechanisms in TAR DNA-binding Protein 43 (TDP-43) Proteinopathies, J Biol Chem 291, 19437-19448.

[9] Griffin, B. A., Adams, S. R., and Tsien, R. Y. (1998) Specific covalent labeling of recombinant protein molecules inside live cells, Science 281, 269-272.

[10] Martin, B. R., Giepmans, B. N., Adams, S. R., and Tsien, R. Y. (2005) Mammalian cellbased optimization of the biarsenical-binding tetracysteine motif for improved fluorescence and affinity, Nat Biotechnol 23, 1308-1314.

[11] Giepmans, B. N., Adams, S. R., Ellisman, M. H., and Tsien, R. Y. (2006) The fluorescent toolbox for assessing protein location and function, Science 312, 217-224.

[12] Roberti, M. J., Bertoncini, C. W., Klement, R., Jares-Erijman, E. A., and Jovin, T. M. (2007) Fluorescence imaging of amyloid formation in living cells by a functional, tetracysteinetagged alpha-synuclein, Nat Methods 4, 345-351.

[13] Hoffmann, C., Gaietta, G., Zurn, A., Adams, S. R., Terrillon, S., Ellisman, M. H., Tsien, R. Y., and Lohse, M. J. (2010) Fluorescent labeling of tetracysteine-tagged proteins in intact cells, Nat Protoc 5, 1666-1677.

[14] Gelman, H., Wirth, A. J., and Gruebele, M. (2016) ReAsH as a Quantitative Probe of In-Cell Protein Dynamics, Biochemistry 55, 1968-1976.

[15] Ignatova, Z., and Gierasch, L. M. (2004) Monitoring protein stability and aggregation in vivo 
by real-time fluorescent labeling, Proc Natl Acad Sci U S A 101, 523-528.

[16] Irtegun, S., Wood, R., Lackovic, K., Schweiggert, J., Ramdzan, Y. M., Huang, D. C., Mulhern, T. D., and Hatters, D. M. (2014) A biosensor of SRC family kinase conformation by exposable tetracysteine useful for cell-based screening, ACS Chem Biol 9, 1426-1431.

[17] Ramdzan, Y. M., Nisbet, R. M., Miller, J., Finkbeiner, S., Hill, A. F., and Hatters, D. M. (2010) Conformation sensors that distinguish monomeric proteins from oligomers in live cells, Chem Biol 17, 371-379.

[18] Roberti, M. J., Jovin, T. M., and Jares-Erijman, E. (2011) Confocal fluorescence anisotropy and FRAP imaging of alpha-synuclein amyloid aggregates in living cells, Plos One 6, e23338, 1-7.

[19] Nonaka, T., Arai, T., Buratti, E., Baralle, F. E., Akiyama, H., and Hasegawa, M. (2009) Phosphorylated and ubiquitinated TDP-43 pathological inclusions in ALS and FTLD-U are recapitulated in SH-SY5Y cells, FEBS Lett 583, 394-400.

[20] Nonaka, T., Masuda-Suzukake, M., Arai, T., Hasegawa, Y., Akatsu, H., Obi, T., Yoshida, M., Murayama, S., Mann, D. M., Akiyama, H., and Hasegawa, M. (2013) Prion-like properties of pathological TDP-43 aggregates from diseased brains, Cell Rep 4, 124134.

[21] Bentmann, E., Neumann, M., Tahirovic, S., Rodde, R., Dormann, D., and Haass, C. (2012) Requirements for stress granule recruitment of fused in sarcoma (FUS) and TAR DNAbinding protein of $43 \mathrm{kDa}$ (TDP-43), J Biol Chem 287, 23079-23094.

[22] Liu-Yesucevitz, L., Bilgutay, A., Zhang, Y. J., Vanderweyde, T., Citro, A., Mehta, T., Zaarur, N., McKee, A., Bowser, R., Sherman, M., Petrucelli, L., and Wolozin, B. (2010) Tar DNA binding protein-43 (TDP-43) associates with stress granules: analysis of cultured cells and pathological brain tissue, Plos One 5, e13250, 1-15.

[23] Fallini, C., Bassell, G. J., and Rossoll, W. (2012) The ALS disease protein TDP-43 is actively transported in motor neuron axons and regulates axon outgrowth, Hum Mol Genet 21, 3703-3718.

[24] Nonaka, T., Kametani, F., Arai, T., Akiyama, H., and Hasegawa, M. (2009) Truncation and pathogenic mutations facilitate the formation of intracellular aggregates of TDP-43, Hum Mol Genet 18, 3353-3364.

[25] Scotter, E. L., Vance, C., Nishimura, A. L., Lee, Y. B., Chen, H. J., Urwin, H., Sardone, V., Mitchell, J. C., Rogelj, B., Rubinsztein, D. C., and Shaw, C. E. (2014) Differential roles of the ubiquitin proteasome system and autophagy in the clearance of soluble and aggregated TDP-43 species, J Cell Sci 127, 1263-1278.

[26] Igaz, L. M., Kwong, L. K., Xu, Y., Truax, A. C., Uryu, K., Neumann, M., Clark, C. M., Elman, L. B., Miller, B. L., Grossman, M., McCluskey, L. F., Trojanowski, J. Q., and Lee, V. M. (2008) Enrichment of C-terminal fragments in TAR DNA-binding protein-43 cytoplasmic inclusions in brain but not in spinal cord of frontotemporal lobar degeneration and amyotrophic lateral sclerosis, Am J Pathol 173, 182-194.

[27] Guo, W., Chen, Y., Zhou, X., Kar, A., Ray, P., Chen, X., Rao, E. J., Yang, M., Ye, H., Zhu, L., Liu, J., Xu, M., Yang, Y., Wang, C., Zhang, D., Bigio, E. H., Mesulam, M., Shen, Y., Xu, Q., Fushimi, K., and Wu, J. Y. (2011) An ALS-associated mutation affecting TDP-43 enhances protein aggregation, fibril formation and neurotoxicity, Nat Struct Mol Biol 18, 822-830.

[28] Peled, S., Sade, D., Bram, Y., Porat, Z., Kreiser, T., Mimouni, M., Lichtenstein, A., Segal, D., and Gazit, E. (2017) Single cell imaging and quantification of TDP-43 and alphasynuclein intercellular propagation, Sci Rep 7, 544, 1-12.

[29] Kovalevich, J., and Langford, D. (2013) Considerations for the Use of SH-SY5Y Neuroblastoma Cells in Neurobiology, Methods Mol Biol 1078, 9-21. 
[30] Limbocker, R., Chia, S., Ruggeri, F. S., Perni, M., Cascella, R., Heller, G. T., Meisl, G., Mannini, B., Habchi, J., Michaels, T. C. T., Challa, P. K., Ahn, M., Casford, S. T., Fernando, N., Xu, C. K., Kloss, N. D., Cohen, S. I. A., Kumita, J. R., Cecchi, C., Zasloff, M., Linse, S., Knowles, T. P. J., Chiti, F., Vendruscolo, M., and Dobson, C. M. (2019) Trodusquemine enhances A beta(42) aggregation but suppresses its toxicity by displacing oligomers from cell membranes, Nat Commun 10.

[31] Liu-Yesucevitz, L., Lin, A. Y., Ebata, A., Boon, J. Y., Reid, W., Xu, Y. F., Kobrin, K., Murphy, G. J., Petrucelli, L., and Wolozin, B. (2014) ALS-linked mutations enlarge TDP-43enriched neuronal RNA granules in the dendritic arbor, J Neurosci 34, 4167-4174.

[32] Dewey, C. M., Cenik, B., Sephton, C. F., Dries, D. R., Mayer, P., 3rd, Good, S. K., Johnson, B. A., Herz, J., and Yu, G. (2011) TDP-43 is directed to stress granules by sorbitol, a novel physiological osmotic and oxidative stressor, Mol Cell Biol 31, 1098-1108.

[33] Feiler, M. S., Strobel, B., Freischmidt, A., Helferich, A. M., Kappel, J., Brewer, B. M., Li, D., Thal, D. R., Walther, P., Ludolph, A. C., Danzer, K. M., and Weishaupt, J. H. (2015) TDP-43 is intercellularly transmitted across axon terminals, J Cell Biol 211, 897-911.

[34] van Eersel, J., Ke, Y. D., Gladbach, A., Bi, M., Gotz, J., Kril, J. J., and Ittner, L. M. (2011) Cytoplasmic accumulation and aggregation of TDP-43 upon proteasome inhibition in cultured neurons, Plos One 6, e22850, 1-13.

[35] Yamashita, M., Nonaka, T., Hirai, S., Miwa, A., Okado, H., Arai, T., Hosokawa, M., Akiyama, H., and Hasegawa, M. (2014) Distinct pathways leading to TDP-43-induced cellular dysfunctions, Hum Mol Gen 23, 4345-4356.

[36] Zhang, Y. J., Xu, Y. F., Cook, C., Gendron, T. F., Roettges, P., Link, C. D., Lin, W. L., Tong, J., Castanedes-Casey, M., Ash, P., Gass, J., Rangachari, V., Buratti, E., Baralle, F., Golde, T. E., Dickson, D. W., and Petrucelli, L. (2009) Aberrant cleavage of TDP-43 enhances aggregation and cellular toxicity, Proc Natl Acad Sci U S A 106, 7607-7612.

[37] Wolozin, B. (2012) Regulated protein aggregation: stress granules and neurodegeneration, Mol Neurodegener 7, 56, 1-12.

[38] Dewey, C. M., Cenik, B., Sephton, C. F., Johnson, B. A., Herz, J., and Yu, G. (2012) TDP43 aggregation in neurodegeneration: are stress granules the key?, Brain Res 1462, 1625.

[39] Higashi, S., Kabuta, T., Nagai, Y., Tsuchiya, Y., Akiyama, H., and Wada, K. (2013) TDP-43 associates with stalled ribosomes and contributes to cell survival during cellular stress, $J$ Neurochem 126, 288-300.

[40] Colombrita, C., Zennaro, E., Fallini, C., Weber, M., Sommacal, A., Buratti, E., Silani, V., and Ratti, A. (2009) TDP-43 is recruited to stress granules in conditions of oxidative insult, $J$ Neurochem 111, 1051-1061.

[41] McGurk, L., Lee, V. M., Trojanowksi, J. Q., Van Deerlin, V. M., Lee, E. B., and Bonini, N. M. (2014) Poly-A binding protein-1 localization to a subset of TDP-43 inclusions in amyotrophic lateral sclerosis occurs more frequently in patients harboring an expansion in C9orf72, J Neuropathol Exp Neurol 73, 837-845.

[42] Li, Y. R., King, O. D., Shorter, J., and Gitler, A. D. (2013) Stress granules as crucibles of ALS pathogenesis, J Cell Biol 201, 361-372.

[43] Inukai, Y., Nonaka, T., Arai, T., Yoshida, M., Hashizume, Y., Beach, T. G., Buratti, E., Baralle, F. E., Akiyama, H., Hisanaga, S., and Hasegawa, M. (2008) Abnormal phosphorylation of Ser409/410 of TDP-43 in FTLD-U and ALS, FEBS Lett 582, 28992904.

[44] Langhorst, M. F., Genisyuerek, S., and Stuermer, C. A. (2006) Accumulation of $\mathrm{FIAsH/Lumio} \mathrm{Green} \mathrm{in} \mathrm{active} \mathrm{mitochondria} \mathrm{can} \mathrm{be} \mathrm{reversed} \mathrm{by} \mathrm{beta-mercaptoethanol} \mathrm{for}$ specific staining of tetracysteine-tagged proteins, Histochem Cell Biol 125, 743-747.

[45] Shiina, Y., Toyoda, T., Kawasoe, Y., Tateno, S., Shirai, T., Matsuo, K., Mizuno, Y., Ai, T., 
and Niwa, K. (2011) The prevalence and risk factors for cholelithiasis and asymptomatic gallstones in adults with congenital heart disease, Int $J$ Cardiol 152, 171-176.

[46] Seyfried, N. T., Gozal, Y. M., Dammer, E. B., Xia, Q., Duong, D. M., Cheng, D., Lah, J. J., Levey, A. I., and Peng, J. (2010) Multiplex SILAC analysis of a cellular TDP-43 proteinopathy model reveals protein inclusions associated with SUMOylation and diverse polyubiquitin chains, Mol Cell Proteomics 9, 705-718.

[47] Budini, M., Romano, V., Avendano-Vazquez, S. E., Bembich, S., Buratti, E., and Baralle, F. E. (2012) Role of selected mutations in the $\mathrm{Q} / \mathrm{N}$ rich region of TDP-43 in EGFP-12xQ/Ninduced aggregate formation, Brain Res 1462, 139-150.

[48] Liu, R., Yang, G., Nonaka, T., Arai, T., Jia, W., and Cynader, M. S. (2013) Reducing TDP43 aggregation does not prevent its cytotoxicity, Acta Neuropathol Commun 1, 49, 1-11.

[49] Walker, A. K., Soo, K. Y., Sundaramoorthy, V., Parakh, S., Ma, Y., Farg, M. A., Wallace, R. H., Crouch, P. J., Turner, B. J., Horne, M. K., and Atkin, J. D. (2013) ALS-Associated TDP-43 Induces Endoplasmic Reticulum Stress, Which Drives Cytoplasmic TDP-43 Accumulation and Stress Granule Formation, Plos One 8, e81170, 1-12.

[50] Hasegawa, M., Arai, T., Nonaka, T., Kametani, F., Yoshida, M., Hashizume, Y., Beach, T. G., Buratti, E., Baralle, F., Morita, M., Nakano, I., Oda, T., Tsuchiya, K., and Akiyama, H. (2008) Phosphorylated TDP-43 in frontotemporal lobar degeneration and amyotrophic lateral sclerosis, Ann Neurol 64, 60-70.

[51] Neumann, M., Kwong, L. K., Lee, E. B., Kremmer, E., Flatley, A., Xu, Y., Forman, M. S., Troost, D., Kretzschmar, H. A., Trojanowski, J. Q., and Lee, V. M. (2009) Phosphorylation of S409/410 of TDP-43 is a consistent feature in all sporadic and familial forms of TDP-43 proteinopathies, Acta Neuropathol 117, 137-149.

[52] Lee, E. B., Lee, V. M., and Trojanowski, J. Q. (2011) Gains or losses: molecular mechanisms of TDP43-mediated neurodegeneration, Nat Rev Neurosci 13, 38-50. 


\section{Using tetracysteine-tagged TDP-43 with a biarsenical dye to monitor real-time trafficking in a cell model of Amyotrophic Lateral Sclerosis}

Janice S. W. $\mathrm{Ng}^{1 * \star}$, Maya A. Hanspal ${ }^{1 * \star}$, Naunehal S. Matharu ${ }^{1}$, Teresa P. Barros ${ }^{1}$, Elin K. Esbjörner $^{2}$, Mark R. Wilson ${ }^{3,4}$, Justin J. Yerbury ${ }^{3,4}$, Christopher M. Dobson ${ }^{1}$ and Janet R. Kumita ${ }^{*}$

${ }^{1}$ Centre for Misfolding Diseases, University of Cambridge, Lensfield Road, Cambridge, CB2 1EW, UK; ${ }^{2}$ Department of Biology and Biological Engineering, Division of Chemical Biology, Chalmers University of Technology, Kemivägen 10, 41296 Gothenburg, Sweden; ${ }^{3}$ lllawarra Health and Medical Research Institute, Wollongong, NSW, Australia; ${ }^{4}$ Molecular Horizons and School of Chemistry and Molecular Bioscience, Faculty of Science Medicine and Health, University of Wollongong, Northfields Avenue, Wollongong, NSW, 2522, Australia.

Running Title: Real-time imaging of TDP-43 trafficking in ALS

*A Authors contributed equally

*To whom correspondence should be addressed: Janet R. Kumita: Centre for Misfolding Diseases, University of Cambridge, Lensfield Road, Cambridge, CB2 1EW, UK, irk38@cam.ac.uk, Tel.+44(0)1223761480 


\section{Supplementary methods}

\section{Transfection efficiency}

SH-SY5Y cells expressing HA-TDP43 or HA-TDP43-TC were fixed and immunostained with anti-HA antibody and nuclear counterstain Hoechst, $24 \mathrm{~h}$ post-transfection (via lipofection or electroporation). These samples were then imaged using confocal microscopy at $40 \mathrm{x}$ magnification, and the number of green (HA-TDP43 or HA-TDP43-TC) and blue (Hoechst) stained cells present in each image were manually counted using the Cell Counter plug-in in ImageJ. The transfection efficiency was calculated as the number of green transfected cells as a percentage of the total number of blue Hoechst-stained cells counted in each image.

\section{Quantification and comparison of nuclear FIAsH fluorescence in cells transfected with} the HA-TDP43-TC plasmid using either lipofection or electroporation

SH-SY5Y cells were transfected with HA-TDP43-TC using both lipofection and electroporation methods and imaged using confocal microscopy $24 \mathrm{~h}$ post-transfection. The nuclear fluorescence in transfected cells was quantified as described in the methods section. A two-tailed unpaired Student t-test was performed on the mean fluorescence for each transfection method using GraphPad Prism version 7 for Mac OS X (GraphPad Software, La Jolla California USA) to determine whether or not there was a significant difference in the intensity of nuclear fluorescence between the two different transfection methods.

\section{Supplementary tables and figures}

Table S1. Oligonucleotide sequences of primers used.

\begin{tabular}{|c|c|}
\hline $\begin{array}{c}\text { Primer } \\
\text { name }\end{array}$ & Sequence \\
\hline $\begin{array}{c}\text { TDP43-TC } \\
\text { (forward) }\end{array}$ & $\begin{array}{c}\text { 5'AATTGGATCCATGTACCCATACGACGTCCCAGACT } \\
\text { ACGCTTCTGAATATATTCGGGTAAC-3' }\end{array}$ \\
\hline $\begin{array}{c}\text { TDP43-TC } \\
\text { (reverse) }\end{array}$ & $\begin{array}{c}\text { 5'-CTAGTCTAGACTAGGGCTCCATGCAACAGCCTGGA } \\
\text { CAGCAGTTCAGGAACATTCCCCAGCCAGAAGACT-3' }\end{array}$ \\
\hline $\begin{array}{c}\text { HA-TDP43 } \\
\text { (forward) }\end{array}$ & 5'-AATTGGATCCATGTACCCATACGACGTCC-3' \\
\hline $\begin{array}{c}\text { HA-TDP43 } \\
\text { (reverse) }\end{array}$ & 5'-CTAGTCTAGAAGCTTCTACATTCCCCAGCCAG-3' \\
\hline
\end{tabular}


Table S2. Transfection efficiency of SH-SY5Y cells transfected with HA-TDP43 or HATDP43-TC plasmid using either lipofection or electroporation

\begin{tabular}{|c|c|c|c|c|}
\hline & $\begin{array}{c}\text { Transfection } \\
\text { method }\end{array}$ & $\begin{array}{c}\text { Number of } \\
\text { green cells }\end{array}$ & $\begin{array}{c}\text { Number of } \\
\text { blue cells }\end{array}$ & $\begin{array}{c}\text { efficiency } \\
(\%)\end{array}$ \\
\hline HA-TDP43 & Lipofection & 264 & 2648 & 9.97 \\
\hline & Electroporation & 53 & 374 & 14.17 \\
\hline HA-TDP43-TC & Lipofection & 236 & 1947 & 12.12 \\
\hline & Electroporation & 44 & 312 & 14.10 \\
\hline
\end{tabular}

Table S3. Nuclear fluorescence values from SH-SY5Y cells expressing HA-TDP43-TC using both lipofection and electroporation methods $24 \mathrm{~h}$ post-transfection.

\begin{tabular}{|c|c|c|}
\hline \multicolumn{3}{|c|}{ HA-TDP43-TC lipofection } \\
\hline Area of measurement & Integrated density & Nuclear fluorescence \\
\hline 92.364 & 2574.874 & 2252.985 \\
\hline 82.204 & 1430.486 & 1144.005 \\
\hline 104.14 & 1566.261 & 1203.333 \\
\hline 96.982 & 1213.431 & 875.449 \\
\hline 63.731 & 1621.448 & 1399.346 \\
\hline 54.957 & 2458.034 & 2266.509 \\
\hline 60.96 & 881.151 & 668.705 \\
\hline 78.059 & 1216.663 & 943.059 \\
\hline Mean background grey value & 3.485 & \\
\hline \multicolumn{3}{|c|}{ HA-TDP43-TC electroporation } \\
\hline Area of measurement & Integrated density & Nuclear fluorescence \\
\hline 78.509 & 1636.226 & 1335.327 \\
\hline 65.347 & 2008.453 & 1757.999 \\
\hline 94.673 & 2441.639 & 2078.789 \\
\hline 111.76 & 1719.123 & 1290.784 \\
\hline 99.522 & 4858.802 & 4477.367 \\
\hline Mean background grey value & 3.833 & \\
\hline
\end{tabular}


A

DNA sequence for HA-TDP43:

AATTGGATCCATGTACCCATACGACGTCCCAGACTACGCTTCTGAATATATTCG GGTAACCGAAGATGAGAACGATGAGCCCATTGAAATACCATCGGAAGACGATG GGACGGTGCTGCTCTCCACGGTTACAGCCCAGTTTCCAGGGGCGTGTGGGCTT CGCTACAGGAATCCAGTGTCTCAGTGTATGAGAGGTGTCCGGCTGGTAGAAGG AATTCTGCATGCCCCAGATGCTGGCTGGGGAAATCTGGTGTATGTTGTCAACTA TCCAAAAGATAACAAAAGAAAAATGGATGAGACAGATGCTTCATCAGCAGTGAA AGTGAAAAGAGCAGTCCAGAAAACATCCGATTTAATAGTGTTGGGTCTCCCATG GAAAACAACCGAACAGGACCTGAAAGAGTATTTTAGTACCTTTGGAGAAGTTCT TATGGTGCAGGTCAAGAAAGATCTTAAGACTGGTCATTCAAAGGGGTTTGGCTT TGTTCGTTTTACGGAATATGAAACACAAGTGAAAGTAATGTCACAGCGACATAT GATAGATGGACGATGGTGTGACTGCAAACTTCCTAATTCTAAGCAAAGCCAAGA TGAGCCTTTGAGAAGCAGAAAAGTGTTTGTGGGGCGCTGTACAGAGGACATGA CTGAGGATGAGCTGCGGGAGTTCTTCTCTCAGTACGGGGATGTGATGGATGTC TTCATCCCCAAGCCATTCAGGGCCTTTGCCTTTGTTACATTTGCAGATGATCAG ATTGCGCAGTCTCTTTGTGGAGAGGACTTGATCATTAAAGGAATCAGCGTTCAT ATATCCAATGCCGAACCTAAGCACAATAGCAATAGACAGTTAGAAAGAAGTGGA AGATTTGGTGGTAATCCAGGTGGCTTTGGGAATCAGGGTGGATTTGGTAATAGC AGAGGGGGTGGAGCTGGTTTGGGAAACAATCAAGGTAGTAATATGGGTGGTGG GATGAACTTTGGTGCGTTCAGCATTAATCCAGCCATGATGGCTGCCGCCCAGG CAGCACTACAGAGCAGTTGGGGTATGATGGGCATGTTAGCCAGCCAGCAGAAC CAGTCAGGCCCATCGGGTAATAACCAAAACCAAGGCAACATGCAGAGGGAGCC AAACCAGGCCTTCGGTTCTGGAAATAACTCTTATAGTGGCTCTAATTCTGGTGC AGCAATTGGTTGGGGATCAGCATCCAATGCAGGGTCGGGCAGTGGTTTTAATG GAGGCTTTGGCTCAAGCATGGATTCTAAGTCTTCTGGCTGGGGAATGTAGAAG CTICTAGACTAG

B

DNA sequence for HA-TDP43-TC:

AATTGGATCCATGTACCCATACGACGTCCCAGACTACGCTTCTGAATATATTCG GGTAACCGAAGATGAGAACGATGAGCCCATTGAAATACCATCGGAAGACGATG GGACGGTGCTGCTCTCCACGGTTACAGCCCAGTTTCCAGGGGCGTGTGGGCTT CGCTACAGGAATCCAGTGTCTCAGTGTATGAGAGGTGTCCGGCTGGTAGAAGG AATTCTGCATGCCCCAGATGCTGGCTGGGGAAATCTGGTGTATGTTGTCAACTA TCCAAAAGATAACAAAAGAAAAATGGATGAGACAGATGCTTCATCAGCAGTGAA AGTGAAAAGAGCAGTCCAGAAAACATCCGATTTAATAGTGTTGGGTCTCCCATG GAAAACAACCGAACAGGACCTGAAAGAGTATTTTAGTACCTTTGGAGAAGTTCT TATGGTGCAGGTCAAGAAAGATCTTAAGACTGGTCATTCAAAGGGGTTTGGCTT TGTTCGTTTTACGGAATATGAAACACAAGTGAAAGTAATGTCACAGCGACATAT GATAGATGGACGATGGTGTGACTGCAAACTTCCTAATTCTAAGCAAAGCCAAGA TGAGCCTTTGAGAAGCAGAAAAGTGTTTGTGGGGCGCTGTACAGAGGACATGA CTGAGGATGAGCTGCGGGAGTTCTTCTCTCAGTACGGGGATGTGATGGATGTC TTCATCCCCAAGCCATTCAGGGCCTTTGCCTTTGTTACATTTGCAGATGATCAG ATTGCGCAGTCTCTTTGTGGAGAGGACTTGATCATTAAAGGAATCAGCGTTCAT ATATCCAATGCCGAACCTAAGCACAATAGCAATAGACAGTTAGAAAGAAGTGGA AGATTTGGTGGTAATCCAGGTGGCTTTGGGAATCAGGGTGGATTTGGTAATAGC AGAGGGGGTGGAGCTGGTTTGGGAAACAATCAAGGTAGTAATATGGGTGGTGG GATGAACTTTGGTGCGTTCAGCATTAATCCAGCCATGATGGCTGCCGCCCAGG CAGCACTACAGAGCAGTTGGGGTATGATGGGCATGTTAGCCAGCCAGCAGAAC CAGTCAGGCCCATCGGGTAATAACCAAAACCAAGGCAACATGCAGAGGGAGCC AAACCAGGCCTTCGGTTCTGGAAATAACTCTTATAGTGGCTCTAATTCTGGTGC AGCAATTGGTTGGGGATCAGCATCCAATGCAGGGTCGGGCAGTGGTTTTAATG GAGGCTTTGGCTCAAGCATGGATTCTAAGTCTTCTGGCTGGGGAATGTTCCTGA ACTGCTGTCCAGGCTGTTGCATGGAGCCCTAGTCTAGACTAG

Figure S1. DNA sequences encoding A) HA-TDP43 and B) HA-TDP43-TC. Restriction enzyme sites (BamHI and Xbal) appear as underlined bold text. The DNA sequence encoding the HA-tag is highlighted yellow in A) and B), and the DNA sequence encoding the TC-tag in B) is highlighted in cyan. 


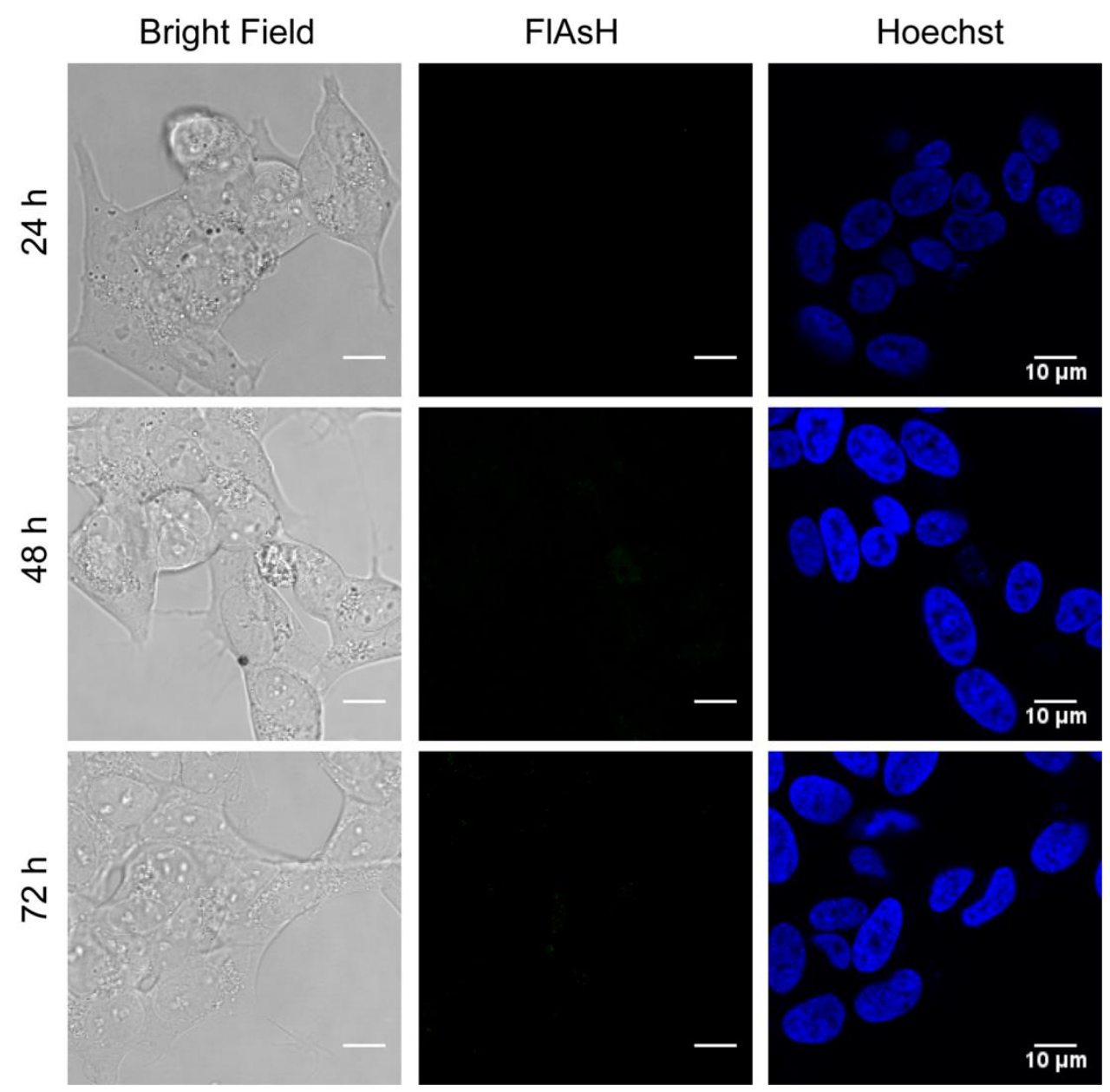

Figure S2. Confocal images showing HA-TDP43 (no TC-tag) in live SH-SY5Y cells at 24, 48 and $72 \mathrm{~h}$ post-transfection in the presence of $\mathrm{FIAsH}$ reagent (middle panels). No FIAsH signal is detected in the absence of the TC-tag. Bright field and Hoechst nuclear counterstain (blue) are shown for each time point. 

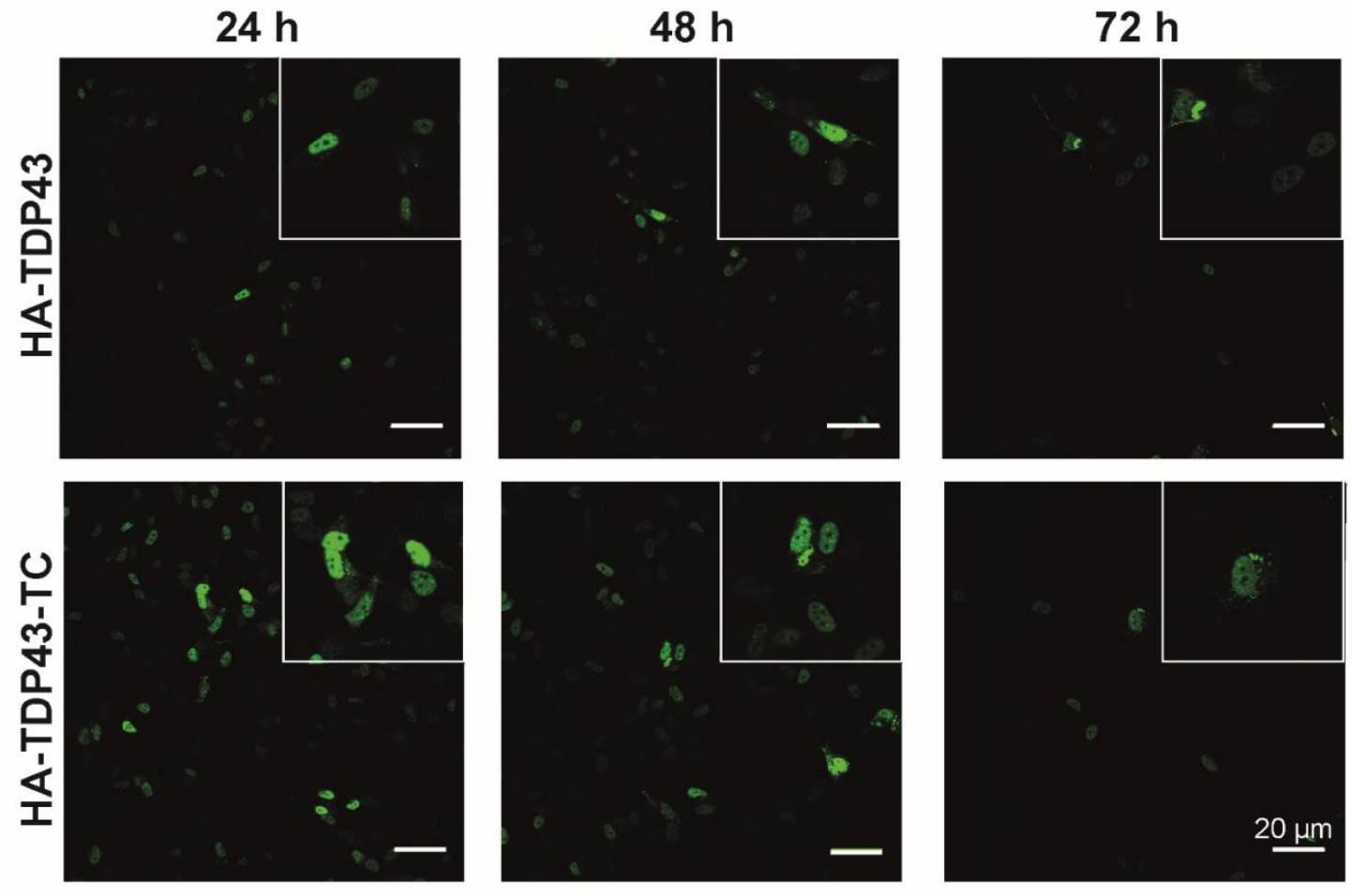

Figure S3. Confocal images showing the distribution of HA-TDP43 and HA-TDP43-TC in lipofected SH-SY5Y cells at 24, 48 and $72 \mathrm{~h}$ post-transfection, immunostained with anti-HA antibody (green). The inset white boxes contain zoomed images of the cell nucleus (zoom factor $2 \times$ original image).
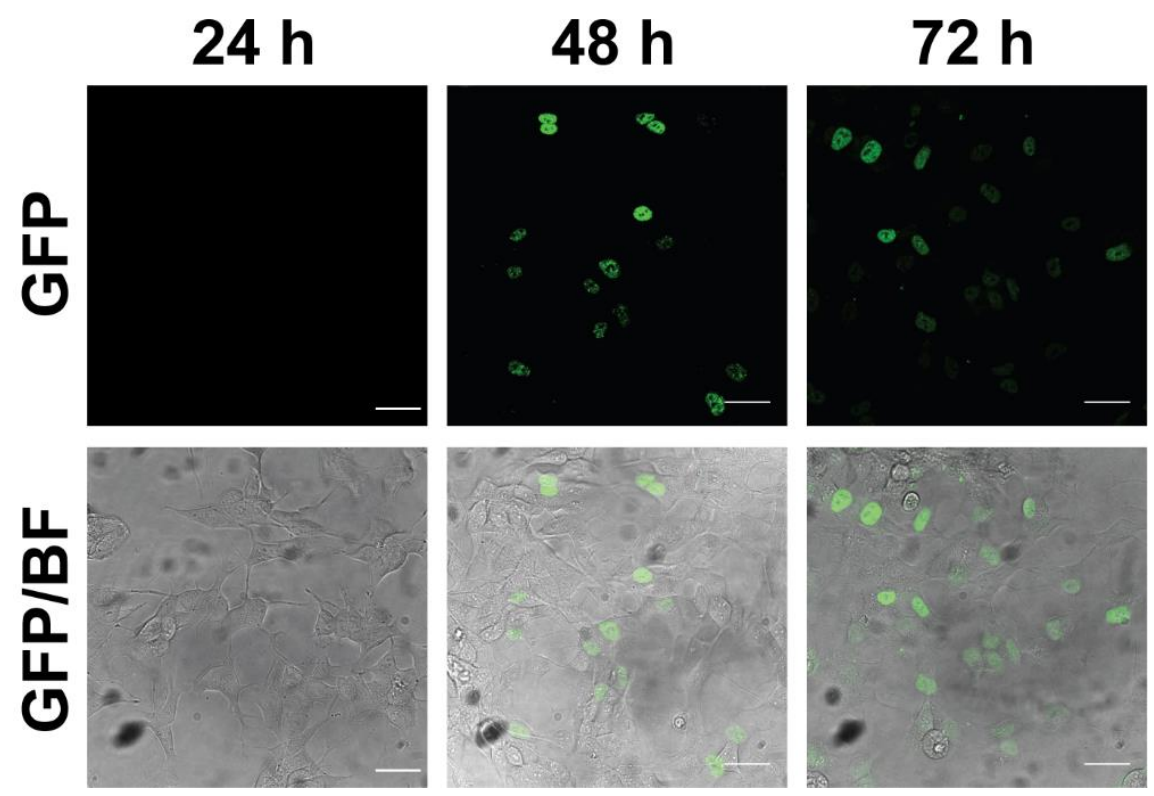

Figure S4. Confocal images showing the distribution of TDP43-GFP (green) in electroporated SH-SY5Y cells at 24, 48 and $72 \mathrm{~h}$ post-transfection. Cells were transfected with a pcDNA4/TO-TDP43-EGFP construct (GenScript, Piscataway, NJ USA) using protocols detailed in the materials and methods. Scale bars are $50 \mu \mathrm{m}$. 


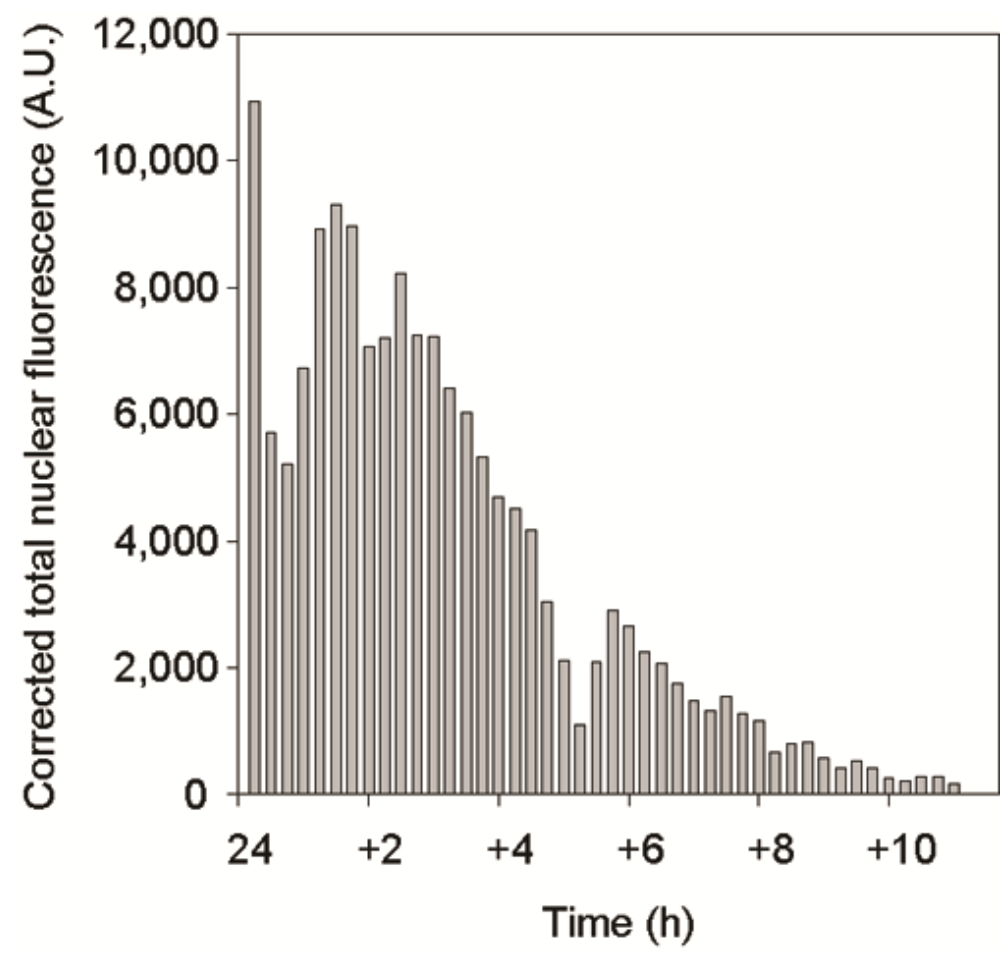

Figure S5. Histogram showing the loss of nuclear fluorescence over $11 \mathrm{~h}$, using HA-TDP43TC expressing SH-SY5Y cells labelled with FIAsH dye $24 \mathrm{~h}$ post-transfection using electroporation. Corrected total nuclear fluorescence was measured using ImageJ and calculated in Excel. The values were obtained from the representative experiment in Figure 7. 


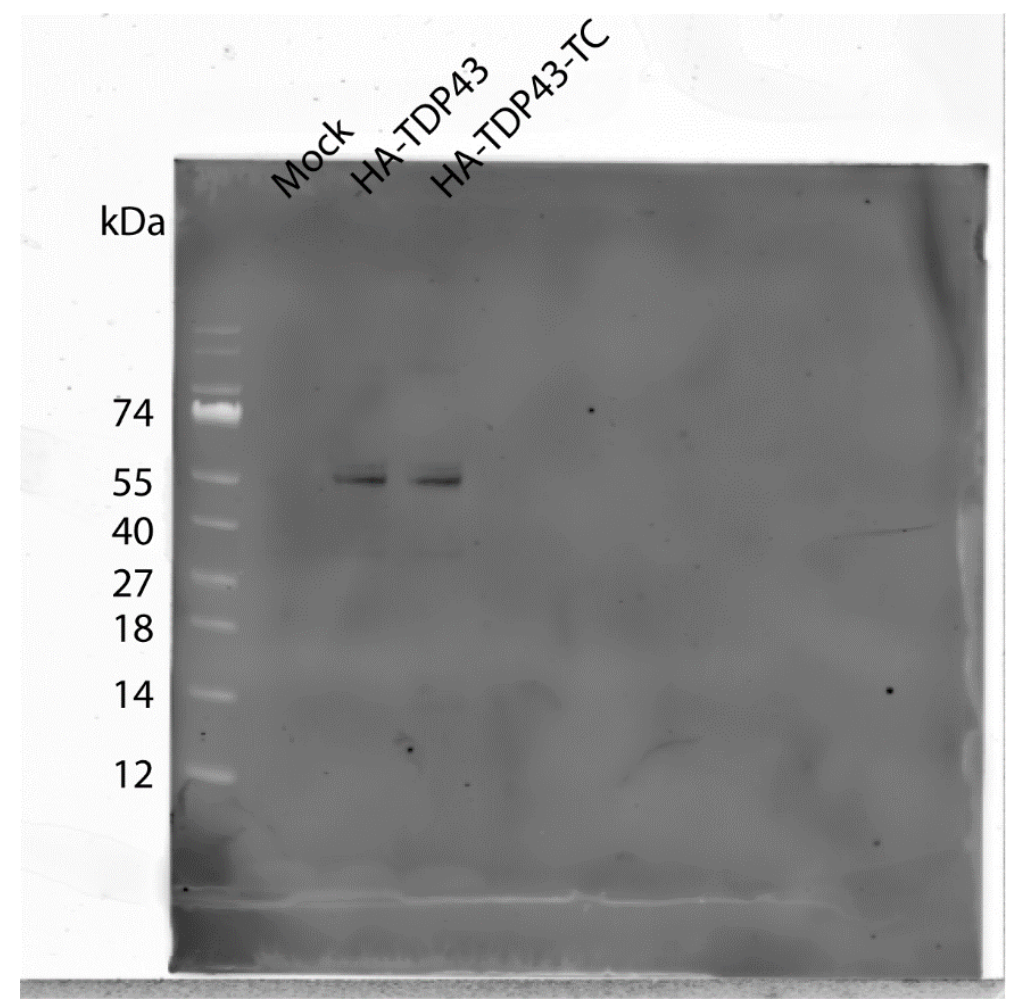

Figure S5. Full western blot from Figure 2, panel C. Western blot analysis following immunoprecipitation purification of HA-TDP43 and HA-TDP43-TC from SH-SY5Y cell lysates at $24 \mathrm{~h}$ post transfection. Mock transfection is cells transfected with buffer alone.

Supplementary Video S1. Time-lapse confocal microscopy video of HA-TDP43-TC expressing $\mathrm{SH}-\mathrm{SY} 5 Y$ cells labelled with FIAsH dye. Imaging commenced at $24 \mathrm{~h}$ posttransfection using electroporation. 